\title{
Serotonin Enhances the Resistance Reflex of the Locomotor Network of the Crayfish through Multiple Modulatory Effects that Act Cooperatively
}

\author{
Morgane Le Bon-Jego, ${ }^{1}$ Daniel Cattaert, ${ }^{1}$ and Edouard Pearlstein ${ }^{2}$ \\ ${ }^{1}$ Laboratoire de Neurobiologie des Réseaux, Centre National de la Recherche Scientifique Unité Mixte de Recherche 5816, Biologie Animale, Bâtiment B2, \\ 33405 Talence cedex, France, and ²Développement et Dysfonctionnement des Réseaux Locomoteurs, Centre National de la Recherche Scientifique, 13402 \\ Marseille cedex 20, France
}

\begin{abstract}
Serotonin $(5 \mathrm{HT})$ is an endogenous amine that modifies posture in crustacea. Here, we examined the mechanisms of action of 5HT on the resistance reflex in crayfish legs. This reflex, which counteracts movements imposed on a limb, is based on a negative feedback system formed by proprioceptors that sense joint angle movements and activate opposing motoneurons. We performed intracellular recordings from depressor motoneurons while repetitively stretching and releasing a leg joint proprioceptor in a resting in vitro preparation (i.e., a preparation that lacks spontaneous rhythmic activity). $5 \mathrm{HT}$ increased the amplitude of the depolarization during the release phase of the proprioceptor (corresponding to an upward movement of the leg) and the discharge frequency of the motoneurons. The 5HT-induced increase in the resistance reflex is caused, to a large extent, by polysynaptic pathways because it was very attenuated in the presence of high divalent cation solution. In addition to this activation of the polysynaptic pathways, $5 \mathrm{HT}$ also has postsynaptic effects that enhance the resistance reflex. 5HT causes a tonic depolarization, as well as an increase in the time constant and input resistance of motoneurons. We developed a simple mathematical model to describe the integrative properties of the motoneurons. The conclusion of this study is that the input frequency and the decay time constant of the EPSPs interact in such a way that small simultaneous changes in these parameters can cause a large effect on summation. Therefore, the conjunction of presynaptic and postsynaptic changes produces a strong cooperative effect on the resistance reflex response.
\end{abstract}

Key words: crustacea; stretch reflex; neuromodulation; posture; serotonin; crayfish

\section{Introduction}

The operation of neural networks controlling behaviors is based on both membrane properties and connectivity of the component neurons. These two levels of a network have been shown to be controlled by neuromodulators in vertebrates and invertebrates (Hultborn and Kiehn, 1992; Katz, 1998; Sillar et al., 1998; Cantrell and Catterall, 2001; Nusbaum et al., 2001; Marder and Thirumalai, 2002). In crustacea, serotonin (5HT) was shown to modify synaptic transmission (Fischer and Florey, 1983; Delaney et al., 1991; Pearlstein et al., 1998; Wang and Zucker, 1998; Southard et al., 2000) and endogenous properties of neurons (Rossi-Durand, 1993; Alvarado-Alvarez et al., 2000; Nagayama, 2002). At the behavioral level 5HT can influence posture and walking (Teshiba et al., 2001; Tierney and Mangiamele, 2001),

Received June 20, 2003; revised 0ct. 21, 2003; accepted 0ct. 21, 2003.

This work was supported by The Centre National de la Recherche Scientifique and by a Groupement d'Intérêt Scientifique (sciences de la cognition) contract (CNA 10). M.L.B.-J. received a fellowship from the Ministère de I'Enseignement Supérieur et de la Recherche. We thank Drs. F. Clarac and P. Meyrand for valuable discussions during the course of this work. We are also grateful to Fadi lssa and Andrew Hill for comments and corrections on this manuscript and for improving the English.

Correspondence should be addressed to D. Cattaert, Laboratoire Neurobiologie des Réseaux, Unité Mixte de Recherche 5816, Université de Bordeaux 1, Centre National de la Recherche Scientifique, Biologie Animale, Bâtiment B2, Avenue des Facultés, 33405 Talence cedex, France. E-mail: d.cattaert@|nr.u-bordeaux.fr.

DOI:10.1523/JNEUROSCI.4032-03.2004

Copyright $\odot 2004$ Society for Neuroscience $\quad$ 0270-6474/04/240398-14\$15.00/0 aggressiveness (Huber et al., 1997a,b), and social status in crayfish (Huber et al., 1997b, 2001). For example, when injected into freely moving lobsters, 5HT and octopamine generate stable and stereotypical postures that resemble those seen in dominant (5HT) and subordinate (octopamine) lobsters (Livingstone et al., 1980; Kravitz, 1988). The analysis of the mechanisms of 5HTinduced neuromodulation is complex because these effects are themselves modulated by the pattern of exposure of the cell to serotonin and the social status of the animal (Edwards et al., 2003).

In this paper, we have studied the effect of $5 \mathrm{HT}$ on postural control in the crayfish Procambarus clarkii. The control of posture involves proprioceptive feedback. In crayfish, the resistance reflex consists of the monosynaptic and polysynaptic excitation of the motoneurons (MNs) that oppose any imposed joint movement (El Manira et al., 1991a,b) and of the polysynaptic inhibition of the antagonistic MNs (Le Bon-Jego and Cattaert, 2002).

In the in vitro preparation of the crayfish walking system (El Manira et al., 1991a), the perfusion of the CNS in a resting preparation (i.e., one that lacks rhythmic activity) with $10 \mu \mathrm{M} 5 \mathrm{HT}$ induces a dramatic increase in the amplitude of the resistance reflex of MNs in most cases. Here, we demonstrate that polysynaptic excitatory pathways of the resistance reflex are activated by $5 \mathrm{HT}$, and we show that $5 \mathrm{HT}$ modifies the intrinsic properties of 
MNs. 5HT causes a depolarization of the resting potential of motoneurons and increases their input resistance and time constant. These multiple effects induced by serotonin act cooperatively to enhance the resistance reflex.

\section{Materials and Methods}

Experimental animals. Experiments were performed on male and female crayfish (Procambarus clarkii; $n=48$ ) weighing $25-30 \mathrm{gm}$. The animals were purchased from a commercial supplier (Château Garreau, La Bastide d'Armagnac, France), maintained in indoor aquaria containing 80 100 animals at $15-18^{\circ} \mathrm{C}$, and fed once a week with mussels.

In vitro preparation. An in vitro preparation of the thoracic nervous system was used (Sillar and Skorupski, 1986; El Manira et al., 1991a). It consists of the last three thoracic (T3-T5) and the first abdominal (A1) ganglia dissected out with all the nerves of the two proximal segments of the left fifth leg (Fig. 1A). The chordotonal organ (CBCO), which monitors the movements of the second joint (coxobasipodite), was also dissected out and kept intact, and the distal end of its elastic strand was attached to an electromagnetic puller VT101 (Ling Dynamic Systems, Meudon-la-Forêt, France) controlled by a homemade function generator allowing the application of sinewave movements to the CBCO strand that mimic upward (during stretch) and downward (during release) movements of the leg. The preparation was pinned dorsal side up on a Sylgard-lined Petri dish (Dow Corning, Wiesbaden, Germany). The nervous system was superfused continuously with oxygenated control saline composed of (in mM): $195 \mathrm{NaCl}, 5 \mathrm{KCl}, 13 \mathrm{CaCl}_{2}, 2 \mathrm{MgCl}_{2}$ and 3 HEPES (Sigma, St Louis, MO) with a $\mathrm{pH}$ of 7.65. The fourth and fifth ganglia were desheathed to improve the superfusion of the central neurons and to allow for intracellular recordings (Fig. $1 A, B$ ). The CNS was insulated from the $\mathrm{CBCO}$ by a Vaseline wall (Fig. $1 A$ ) to restrict the superfusion of 5HT $(10 \mu \mathrm{M})$ to the ganglia. At the same time the $\mathrm{CBCO}$ was superfused with control saline. In some experiments, a high divalent cation solution was used to raise the spiking threshold of the interneurons containing (in $\mathrm{mm}$ ): $34 \mathrm{CaCl}_{2}, 6.4 \mathrm{MgCl}_{2}$, with sodium concentration reduced accordingly to preserve the osmolarity of the solution $(157 \mathrm{NaCl})$.

Recordings. Extracellular recordings from the motor nerves innervating the depressor (Dep) and levator (Lev) muscles and from the sensory nerve of the $\mathrm{CBCO}$ were made using stainless steel pin electrodes contacting the nerves and insulated with Vaseline. Intracellular recordings from depressor MNs (Fig. 1B, Dep MN) were performed with glass micropipettes (Clark Electromedical Instruments, Reading, UK) filled with $3 \mathrm{M} \mathrm{KCl}$ (resistance 10-20 M 2 ) connected to an Axoclamp 2B amplifier (Axon Instruments, Foster City, CA) used in the current-clamp mode. In some experiments, the injection of fluorescent dye (5\% dextran rhodamine in $0.2 \mathrm{M}$ potassium acetate) was used to stain and analyze the geometry of the recorded MN after the experiment (Fig. $1 B$ ). In crustacean MNs, the soma do not participate in the electrical activity of the neuron. $\mathrm{MN}$ soma lie outside of the neuropile (the region in which neurons form their synaptic contacts) (Fig. 1B) and are linked to the arbor of the neuron by a thin neurite. For these reasons, intracellular recordings were made from the main neurite, where EPSPs could be recorded (Fig. $1 B$ ). The resting membrane potential of MNs was usually in the range of -78 to $-65 \mathrm{mV}$. Stability of resting membrane potential over long period of time ( $>4 \mathrm{hr}$ ) was used as a criterion for evaluation of cell health in recordings. In crustacean MNs, the soma and neurites do not actively convey spikes. Therefore, spike amplitude was generally small $(<20 \mathrm{mV})$ at the recording site. Data were digitized and stored onto a computer hard disk through an appropriate interface (1401plus) and software (Spike2) from Cambridge Electronic Design Ltd. (Cambridge, $\mathrm{UK})$.

Data analysis. Data were analyzed using the Spike2 analysis software. Spikes recorded from the CBCO nerve were discriminated according to their waveform on the basis of a template matching protocol (wavemark). Templates were built automatically and corresponded to the mean duration of sensory spikes ( $\sim 1.5 \mathrm{msec}$ in duration). The sampling rate for $\mathrm{CBCO}$ nerve recording was set to $15 \mathrm{kHz}$, which resulted in templates containing $20-22$ points. The procedure used two criteria to identify a spike: (1) $>90 \%$ of the points should be in the confidence
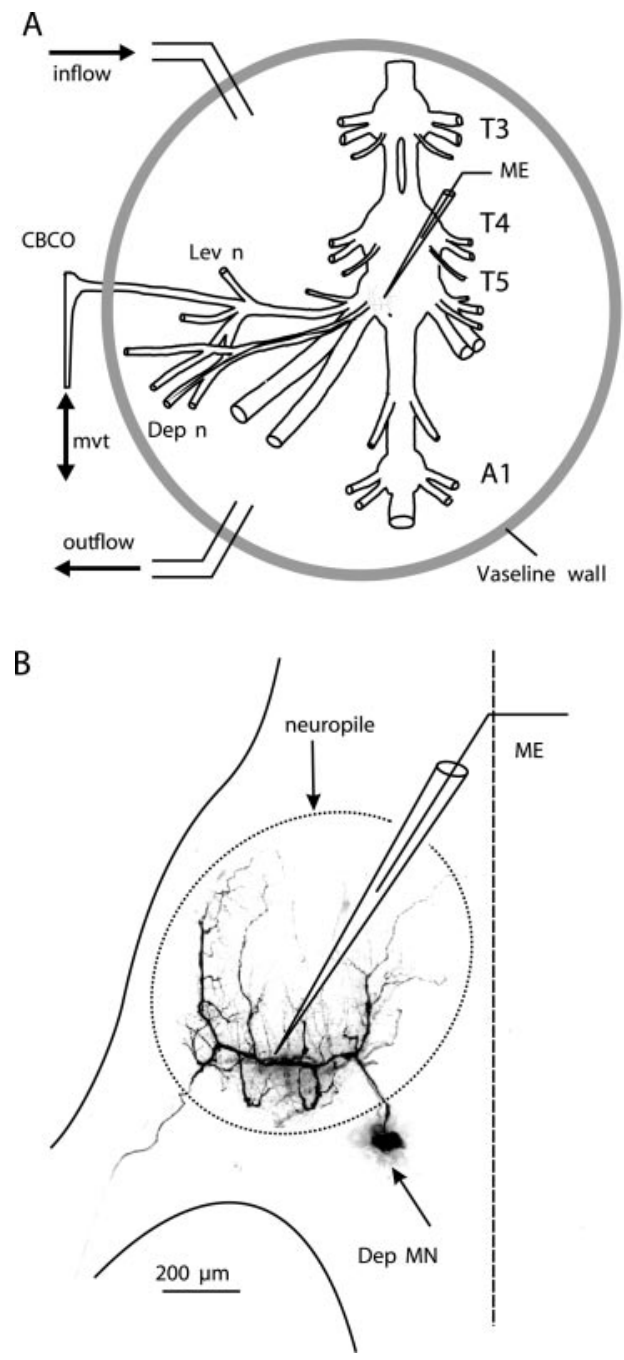

Figure 1. In vitro preparation for the study of the resistance reflex of the second leg joint of the crayfish. $A$, The in vitro preparation of the crayfish thoracic locomotor system consists of thoracic ganglia 3-5 (T3, T4, T5) and the first abdominal ganglion (A1) dissected out together with motor nerves of the proximal muscles (Lev; Dep) and the $\mathrm{CBCO}$, a proprioceptor that encodes the vertical movements of the leg. A mechanical puller allowed us to mimic the vertical movements (mvt) of the leg by stretching and releasing the $\mathrm{CBCO}$ strand. The CNS was isolated from the $\mathrm{CBCO}$ by a Vaseline wall to superfuse only the ganglia with $5 \mathrm{HT}(10 \mu \mathrm{m})$. Single or multiple intracellular recordings from motoneurons and interneurons were performed within the neuropile with glass microelectrodes (ME). $B$, Intracellular staining of a Dep MN. This MN was filled with Dextran rhodamine and analyzed with the confocal microscope. The recording microelectrode (ME) was placed in the main neurite. The neuropile of the ganglion is indicated with a dotted line.

limits of the template; (2) the maximum amplitude change for a match was $<5 \%$. This procedure was applied off-line. After the completion of this protocol, each identified CBCO unit (spike shape) was assigned an arbitrary number. Subsequently, a spike-triggered average was performed for each CBCO unit, allowing us to observe in a given MN the occurrence of any postsynaptic events related to this unit. Statistical analyses were done with the Prism program (Graphpad Software, San Diego, $\mathrm{CA})$. The results are given as mean values \pm SEM.

\section{Results}

5HT enhances resistance reflex

Application of stretch and release movements to the CBCO strand in the in vitro preparation results in reflex activation of MNs (El Manira et al., 1991a,b; Le Ray et al., 1997a,b). During sinewave movements applied to the CBCO strand, Dep and Lev 


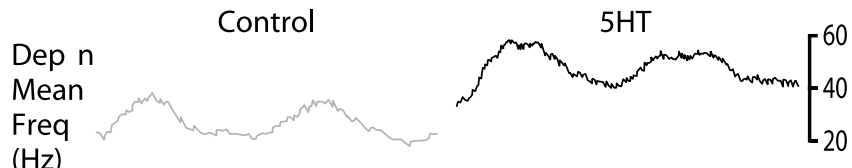

$(\mathrm{Hz})$
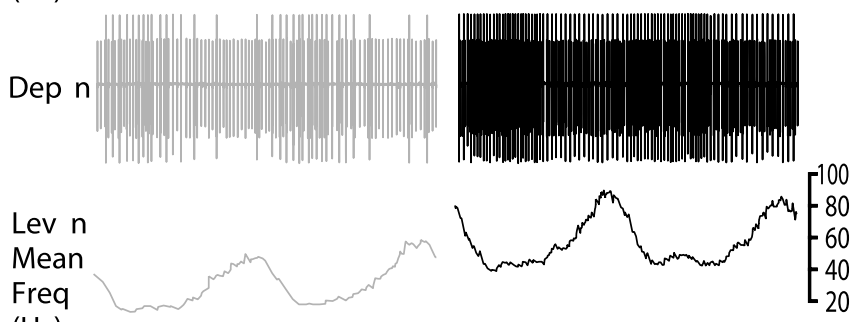

$(\mathrm{Hz})$

Lev $n$
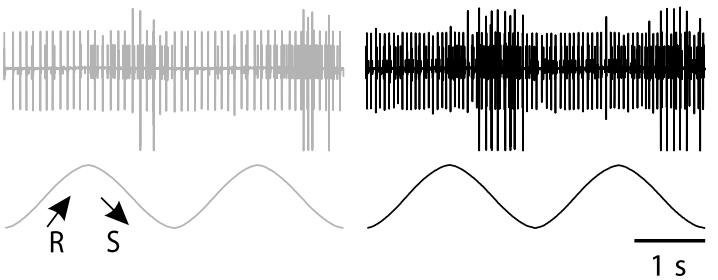

mvt

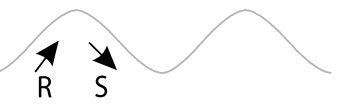

1

Figure 2. Effect of $5 \mathrm{HT}$ on the resistance reflex in a resting preparation. Sinusoidal movements imposed on the $\mathrm{CBC} 0$ strand induced resistance reflex responses recorded extracellularly from the levator nerve (Lev $n$ ) and the depressor nerve (Dep n). When the CBCO strand was released ( $R$; mimicking upward movement in the intact animals), the Dep MNs were activated. In contrast, during stretch movements (S), the Lev MNs were activated. The mean frequency of the discharge of the motor activity recorded is presented above each recording (Dep $n$ Mean Freq, Lev $\mathrm{n}$ Mean Freq). The mean frequency was calculated by a floating average with a time window of $100 \mathrm{msec}$. When $5 \mathrm{HT}$ was superfused on the ganglia, the resistance reflex response of Dep and Lev MNs was increased.

MNs were cyclically activated: Dep MNs during release (mimicking upward movement of the leg in intact animals) and Lev MNs during stretch (mimicking downward movements of the leg in intact animals). These responses corresponded, therefore, to resistance reflex (Fig. 2). All the experiments reported here $(n=48)$ were made in the absence of rhythmic activity. In such preparations, MNs generally display passive properties: their VI curve is linear (at least in the range of a few millivolts from their resting potential) (Le Ray et al., 1997a). On these preparations, we have tested the effects of $10 \mu \mathrm{M} 5 \mathrm{HT}$, a concentration that was shown to produce significant effects both in peripheral sensory organs (Rossi-Durand, 1993) and in the CNS (Pearlstein et al., 1998). When $5 \mathrm{HT}(10 \mu \mathrm{M})$ was superfused on the ganglia $(1 \mathrm{ml} / \mathrm{min})$, two effects were observed on motor nerve activity: (1) the tonic background activity of MNs increased in both Dep and Lev MNs (see mean frequency traces in Fig. 2), and (2) the resistance reflex response of Dep and Lev MNs was increased. This increase was a result of previously silent $\mathrm{MNs}$ being recruited in the presence of $5 \mathrm{HT}$. Moreover, the discharge frequency of previously active MNs increased (this effect was general and is particularly obvious for the large unit in levator nerve activity) (Fig. 2).

In intracellular recordings from MNs (Fig. 3, Dep MN), 5HT induced two kinds of effects: (1) a slow tonic depolarization and (2) an increase in the amplitude of the reflex response. The slow tonic depolarization was observed in 40 experiments. It started $\sim 1$ min after application of $5 \mathrm{HT}$ and reached a maximum of $2-4$ $\mathrm{mV}$ within $<5 \mathrm{~min}$ (Fig. $3 A$ ). This tonic depolarization was independent of movement response (data not shown). The amplitude of the reflex response was measured from averaged traces from a base level (the mean membrane potential between two volleys of EPSPs) to the peak membrane potential during the volley of EPSPs. Note that the amplitude of the reflex response
A

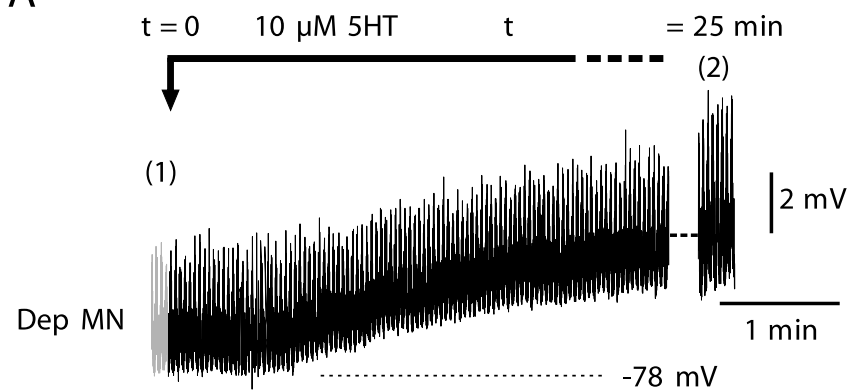

B

Control (1)

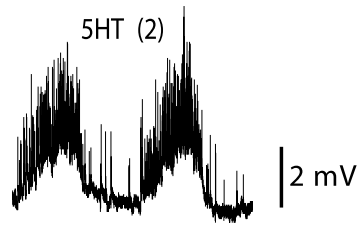

$-78 \mathrm{mV}$

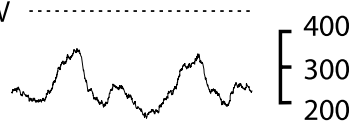

$(\mathrm{Hz})$

$\mathrm{CBCO} \mathrm{n}$

mvt

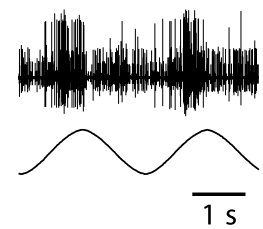

Figure 3. Effect of 5HT on intracellularly recorded Dep MNs. A, After application of 5HT (10 $\mu \mathrm{M})$, an intracellularly recorded Dep MN displays a slight tonic depolarization. During this recording, sinewave movements were applied continuously to the $\mathrm{CBCO}$ strand and evoked a resistance reflex in the Dep MN (see enlarged view in $B$ ). The amplitude of this reflex response was increased after $25 \mathrm{~min}$ in the presence of $5 \mathrm{HT}$. $B$, Enlarged views of recordings presented in $A$. The numbers 1 and 2 refer to the control situation ( $A$, control; $t=0$ ) and to 25 min after onset of $5 \mathrm{HT}$ perfusion $(A, 5 \mathrm{HT} ; t=25 \mathrm{~min})$. This increase in the amplitude of the resistance reflex response was not caused by any change in the sensory activity from the $C B C O$ nerve as demonstrated by the mean frequency of discharge of the sensory nerve of the $\mathrm{CBCO}$ ( $\mathrm{CBCO} \mathrm{n}$ Mean Freq). The resting potential in control situation is indicated by a dashed line $(-78 \mathrm{mV})$ in $A$ and B.

includes both a reflex depolarization (El Manira et al., 1991a; Le Ray et al., 1997b) and a reflex hyperpolarization of Dep MN membrane potential caused by resistance reflex inhibitory pathways (Le Bon-Jego and Cattaert, 2002). In the presence of 5HT, the amplitude of the resistance reflex response increased from $2.23 \pm 0.45 \mathrm{mV}(n=10$ cycles $)$ in control situation to $5.21 \pm 0.12$ $\mathrm{mV}(n=10$ cycles) after $25 \mathrm{~min}$ in the presence of $5 \mathrm{HT}$ (Fig. $3 B$ ). More generally, the increase in the amplitude of the resistance reflex response under 5HT condition, was observed in 58 Dep MNs (from 40 experiments), and the maximum effect observed after 25 min ranged from 8 to $358 \%$.

This increase of the amplitude of the reflex response was not a result of a change in the sensory activity of the CBCO. Sensory activity remained identical for the duration of an experiment as demonstrated by the $\mathrm{CBCO}$ nerve mean frequency discharge (Fig. $3 B$ ). The precaution of building a Vaseline wall to confine the perfusion of $5 \mathrm{HT}$ to the CNS (Materials and Methods) was essential because when $5 \mathrm{HT}$ is applied on the CBCO proprioceptor, the response of sensory neurons to mechanical stimulation of the CBCO strand is greatly enhanced (Rossi-Durand, 1993). To ensure that no leakage occurred, we used two procedures. (1) $5 \mathrm{HT}$ was applied in a mixture containing fast green to get a visual 
A

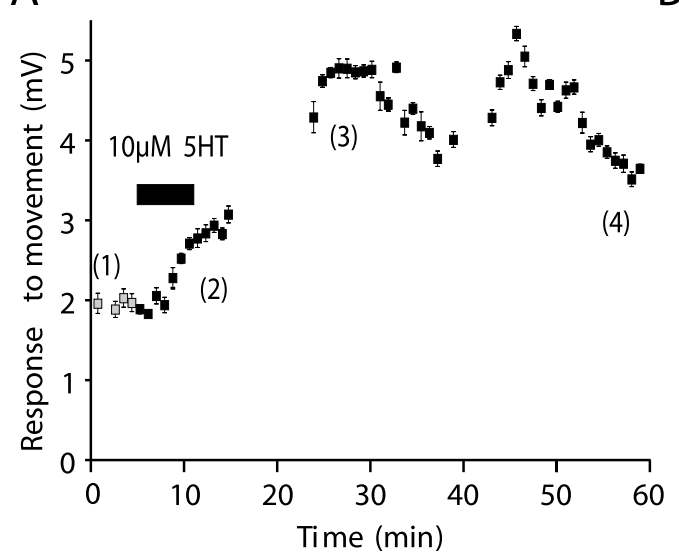

B

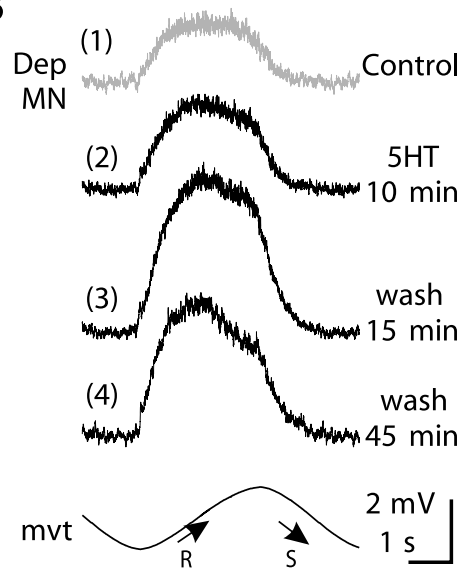

Figure 4. Time course of $5 \mathrm{HT}$ effect on the resistance reflex response. $A$, Amplitude of the intracellular resistance reflex responses of a Dep MN to sinewave movement imposed on the $\mathrm{CBCO}$ strand plotted against time. Each point is an average of the amplitude of 10 movement cycles. Error bars represent SEM. 5HT exposure is indicated by a gray bar. $B$, Average traces $(n=10$ cycles) in four conditions: control (1), 5 HT $10 \mathrm{~min}$ (2), wash $15 \mathrm{~min}$ (3), and wash $45 \mathrm{~min}$ (4). The 5 HT-induced increase in the amplitude of the resistance reflex response persists over 45 min of washing.

A control

Dep MN 1

$\mathrm{mvt}$

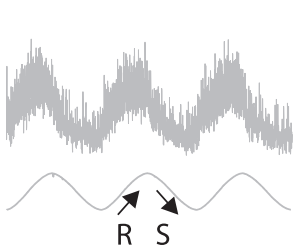

B

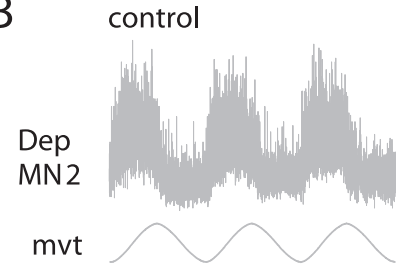

$25 \min 10 \mu \mathrm{M} 5 \mathrm{HT}$

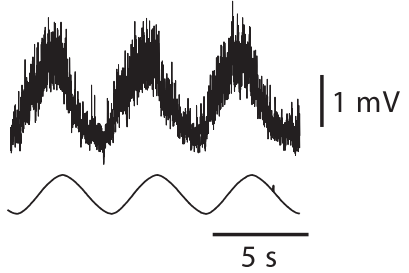

$25 \min 10 \mu \mathrm{M} 5 \mathrm{HT}$

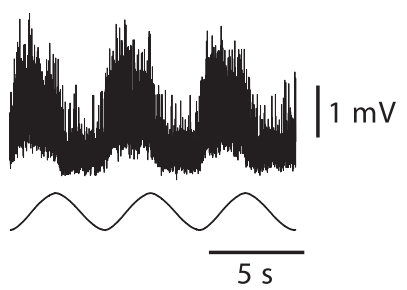

Figure 5. Diversity of $\mathrm{MN}$ response to $5 \mathrm{HT} . A, B$, Raw data of two intracellularly recorded Dep MNs ( $A$, Dep MN 1; B, Dep MN 2) showing resistance reflex responses to a sinewave movement imposed on the $\mathrm{CBC} O \mathrm{in}$ two experiments before (left, control) and after (right, $25 \mathrm{~min} 5 \mathrm{HT}$ ) $5 \mathrm{HT}$ exposure. Although $5 \mathrm{HT}$ induces an increase in Dep MN1 reflex response, $5 \mathrm{HT}$ has no effect on the intracellularly recorded Dep MN2. Resting membrane potentials in control situation were -78 and $-62 \mathrm{mV}$ for Dep MN1 and Dep MN2, respectively. In the presence of 5HT, Dep MN1 showed a tonic depolarization of $3 \mathrm{mV}$ (not visible because traces are aligned for better appreciation of the enhancement of the response in the presence of $5 \mathrm{HT}$ ). No tonic depolarization was observed in Dep MN2 in the presence of 5HT.

confirmation of the absence of leakage, and (2) the mean frequency of the global sensory discharge recorded from the CBCO sensory nerve was monitored to ensure that it did not change during an experiment (Fig. 3B).

\section{The enhancement of the $\mathrm{MN}$ resistance reflex by $5 \mathrm{HT}$ is long lasting}

The two effects produced by 5HT application on Dep MN (slight tonic depolarization and an increase in the amplitude of the resistance reflex response) occurred with different time courses. In the presence of $5 \mathrm{HT}$, although the small tonic depolarization started after $\sim 1$ min (Fig. $3 A$ ), the increase in the amplitude of the reflex response was not observed before 5-10 $\mathrm{min}$ in the

presence of 5HT (Fig. $4 A$ ) at a time when the tonic depolarization was maximum. In Figure $4 A$, the intracellular responses to sinewave movements imposed on the CBCO strand were averaged by groups of 10 cycles (Fig. $4 A, B$ ), and the error bars represent the SEM. In the control situation, the amplitude of the reflex response was $1.94 \pm 0.32 \mathrm{mV}(n=10$ cycles $)$. The amplitude of the response increased to $3.95 \pm 0.33 \mathrm{mV}(n=10$ cycles $)$ after 10 min in the presence of 5HT, and the maximum reflex response was $4.88 \pm 0.32 \mathrm{mV}$ ( $n=10$ cycles) after $25 \mathrm{~min}$ in the presence of 5HT. Therefore, 5HT application caused the response to $\mathrm{CBCO}$ movement to increase by $151 \%$. This effect persisted for $>1 \mathrm{hr}$ (Fig. $4 A, B$ ). When an effect of 5 HT on resistance reflex was observed $(n=40$; see below), its time course was very consistent and more or less similar to the experiment presented in Figure $4 A$, regardless of the 5HT application protocol used (long application $>1$ hr to short $10 \mathrm{~min}$ applications). Note that in this series of experiments, the rate of the perfusion remained unchanged (1 $\mathrm{ml} / \mathrm{min}$ ). In all cases, the reflex response increased progressively after a delay of 5-10 min, and reached a plateau 25 min after the beginning of $5 \mathrm{HT}$ application, and persisted for $>1 \mathrm{hr}$ after washing. Therefore, in all subsequent experiments, we applied 5HT for $10 \mathrm{~min}$ and measured its effects during $1 \mathrm{hr}$ (as in Fig. 4). Subsequently, in all of the figures and in all the following text, the expression "after 5HT application" will mean that the presented effect of 5HT was measured after $25 \mathrm{~min}$ in the presence of 5HT, at a time during which the effect was maximum.

In the experimental conditions used, generally all Dep MNs were silent in the Dep motor nerve. In this paper, only Dep MNs with naturally hyperpolarized resting membrane potentials are presented (Figs. 4, 6A). The spike threshold $(-55$ to $-50 \mathrm{mV}$ ) was never reached in these MNs in response to imposed CBCO movements even after 5HT application. This allowed us (1) to measure continuously the change in response to movement and the input resistance of MNs without contamination by sodium and potassium conductances supporting spikes and (2) to analyze the changes in EPSP amplitude (see below). Dep MNs that responded to $\mathrm{CBCO}$-imposed movements by bursts of spikes in the experimental conditions were generally rare (fewer than two per preparation).

\section{Diversity of $\mathrm{MN}$ response to $5 \mathrm{HT}$}

Although 5HT increased the resistance reflex response in most experiments, in $16.6 \%$ of the 48 experiments, 5 HT did not produce any effect on the recorded MN. The absence of effect was not correlated with the amplitude of the control reflex response: among the experiments in which 5HT had no effect on the intracellularly recorded MNs, we found MNs with either small or large reflex responses in control situations. Figure 5 presents two experiments. In the control situation, a sinewave movement imposed on the CBCO strand elicited a cyclic resistance reflex response in the two Dep MNs (Fig. 5A,B, left). The mean amplitude of the response was $1.81 \pm 0.04 \mathrm{mV}$ ( $n=16$ cycles $)$ in Dep MN1 and $0.87 \pm 0.04 \mathrm{mV}(n=11$ cycles $)$ in Dep MN2. At $t=0$, the preparation was bathed in $10 \mu \mathrm{M} 5 \mathrm{HT}$. At $t=10 \mathrm{~min}$, the preparation was washed in normal Ringer's solution. The 
effect of 5HT on Dep MN1 was maximum at $t=25 \mathrm{~min}$ : its response increased twofold (2.59 $\pm 0.06 \mathrm{mV} ; n=16$ ) (Fig. $5 A$, right). By contrast, in the other experiment, Dep MN2 did not show any significant change ( $t$ test, $p=0.39)$ in its response (0.82 $\pm 0.05 \mathrm{mV} ; n=11)$ (Fig. $5 B$, right). Such a discrepancy between recorded MNs could indicate either a difference between MNs or a difference between the preparations.

To clarify this point, we performed simultaneous intracellular recordings from up to four Dep MNs in a given experiment. In Figure 6, four Dep MNs were intracellularly recorded simultaneously. All of them displayed a cyclic resistance reflex response during sinewave movement applied to the CBCO strand. As in Figure 3, after $1 \mathrm{~min}$ in the presence of $5 \mathrm{HT}$, these four Dep MNs showed a small tonic depolarization (Fig. 6A), and the amplitude of the movement response increased to reach a maximum after $25 \mathrm{~min}$ (Fig. $6 \mathrm{~A}$, right). Superimposition of control and 5HT (25 min) responses indicates that the four MNs displayed an increased response under 5HT condition; however, the magnitude of the effects was different (8-98\%) among the different recorded MNs (Fig. $6 A$, right). In addition to the intracellularly recorded Dep MNs, other Dep MNs showed an increase of their reflex response as monitored by the progressive increase of their reflex discharge recorded from the depressor nerve. During the experiment, application of 5HT did not affect the activity of CBCO sensory neurons as attested by the enlarged view of the CBCO nerve recording (Fig. $6 \mathrm{~B}$ ). Similar results were obtained in five other experiments.

By contrast, in other experiments, 5HT did not induce any changes in all of the simultaneous recordings from four Dep MNs (Fig. 7). It is noteworthy that in such experiments $(n=4)$, neither the small tonic depolarization nor the slower increase in the movement response was observed.

Remarkably, in no experiment in which four simultaneous intracellular recordings from Dep MNs involved in resistance reflex were performed was a combination of 5HT-sensitive and 5HTinsensitive Dep MNs observed. Therefore, it seems that the preparations could be classified into two categories: the ones in which $5 \mathrm{HT}$ induced a general increase in the reflex response in all Dep MNs and the ones in which 5HT did not induce any change in any of the Dep MNs. Hereafter, we have focused on experiments in which 5 HT induced an increase in the resistance reflex response.

\section{HT increases input resistance in Dep MNs}

We have tested the idea that the increase in the response could be caused by a direct effect of 5HT on the MNs. Therefore, during subsequent experiments, we made continuous measurements of the input resistance of intracellularly recorded Dep MNs. Two examples of such experiments are shown in Figure 8. In one of the experiments (Fig. $8 A, C$, Dep MN1), the resistance reflex response was $1.25 \pm 0.26 \mathrm{mV}$ in the control situation and reached $1.65 \pm 0.34 \mathrm{mV}$ after $5 \mathrm{HT}$ application. In the same Dep MN1, the input resistance that was $2.05 \pm 0.15 \mathrm{M} \Omega$ in control situation increased to $2.46 \pm 0.22 \mathrm{M} \Omega$ after $5 \mathrm{HT}$ application. In another experiment, the amplitude of the response of a Dep MN (Fig. 8 B, Dep MN2) was $1.96 \pm 0.15 \mathrm{mV}$ in the control situation and reached $4.12 \pm 0.34 \mathrm{mV}$ after $5 \mathrm{HT}$ application. Accordingly, the input resistance of Dep MN2 increased from 2.16 $\pm 0.12 \mathrm{M} \Omega$ in the control situation to $5.83 \pm 0.16 \mathrm{M} \Omega$ after $5 \mathrm{HT}$ application (Fig. $8 D$ ).

The increase in input resistance was generally in accordance with the increase in the amplitude of the resistance reflex response. For example a moderate increase in movement response (32\% in the case of Dep MN1) corresponded with a moderate increase (20\%) in input resistance. Similarly, a large increase in movement response $(110 \%$ in the case of Dep MN2) corresponded with a large increase in input resistance (169\%). These 


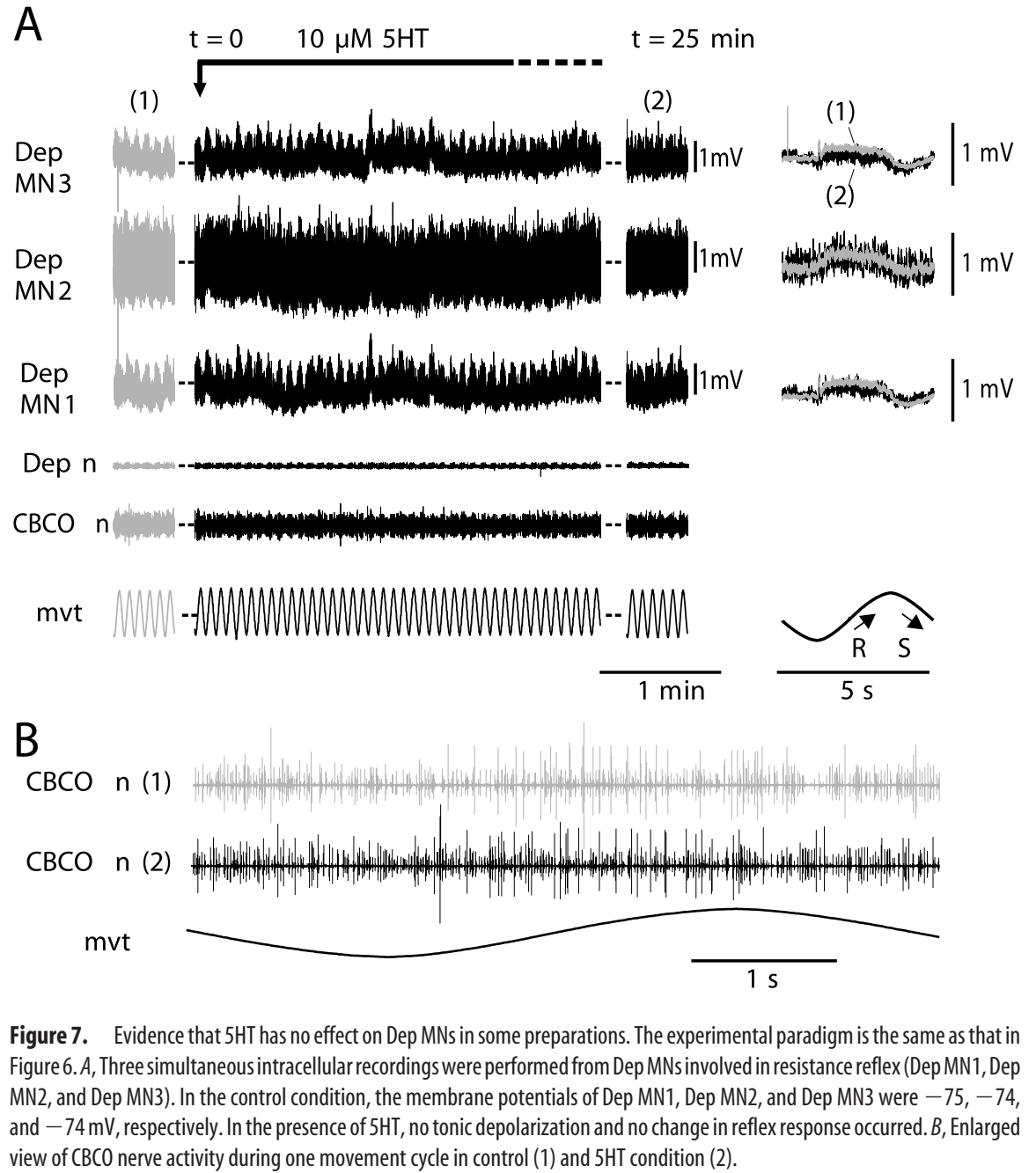

(gray trace) and 5HT (black trace) conditions. With each unitary EPSP, the shape of the corresponding sensory spike (Unit) is presented, together with the histogram of its firing activity during the sinewave movement imposed on the CBCO strand. As can be seen, these sensory units fired during release movement of the $\mathrm{CBCO}$ strand (i.e., upward movement of the leg in intact animals), and because they evoked an EPSP in a Dep MN, they therefore were involved in a resistance reflex. Note that some sensory units were also slightly activated during the stretch movement imposed on the CBCO strand (corresponding to downward movement of the leg). During the downward movement, Dep MNs receive IPSPs via a polysynaptic pathway (Le Bon-Jego and Cattaert, 2002). These IPSPs reduce the amplitude of the unitary EPSPs. Therefore the analysis of unitary EPSP amplitude was performed exclusively during the upward movements. The most remarkable result of this analysis was that the amplitude of unitary EPSPs was only slightly affected if at all in the presence of 5HT (Table 1). For six of eight resistance reflex units, the unitary EPSP was either unchanged (units 13, 15 and $21 ; t$ test; $p>0.2$ ) or even slightly but significantly reduced (units 12,17 and 27; $t$ test; $p<0.0001, p=0.0008, p=$ 0.026 , respectively) in the presence of $5 \mathrm{HT}$ (Fig. 9B, Table 1). This result was very surprising because in the presence of $5 \mathrm{HT}$ the input resistance was increased almost observations were consistent over the eight Dep MNs tested in this series of experiments.

This result was confirmed further by measuring the repolarization time constant in control and 5HT conditions (Fig. 8E,F). In each of the eight tested Dep MNs, the time constant was significantly increased after 5HT application. This increase was highly correlated with the increase in the movement response (Fig. 8G). Similarly, the increase in input resistance was also correlated with the increase in movement response (Fig. 8G). Therefore, it was very likely that the increase in the movement response was directly related to the increase in input resistance of the recorded Dep MN. To confirm this hypothesis, however, we analyzed the effect of 5HT on the amplitude of unitary EPSPs evoked by identified CBCO sensory neurons.

\section{Effect of 5HT on unitary EPSPs from CBCO sensory neurons} The extracellular recording of the CBCO nerve was analyzed by Spike2 software to separate the different sensory units according to their shape. Then each spike shape was used to perform spiketriggered averaging of the intracellular recording of the Dep MN. In Figure 9, only the spikes that were associated with an EPSP in the Dep MN are shown. Each of the spike shapes was monitored continuously during an experiment. In this experiment, after 5HT application, the movement response increased from $0.78 \pm$ 0.08 to $1.52 \pm 0.09 \mathrm{mV}$ (95.3\% increase) (Fig. 9A). At the same time, eight unitary EPSPs triggered by identified sensory units were analyzed (Fig. $9 B, C$ ). Each unitary EPSP is shown in control fourfold, leading us to expect a proportional increase in the amplitude of individual EPSPs. Indeed, only two resistance reflex units (the two with the largest spike amplitudes) presented a net increase of EPSP amplitude under 5HT application (Fig. 9C). These two EPSPs were characterized by complex shapes: an initial rapid EPSP followed by a second slower depolarizing wave. The detailed analysis of one of these EPSPs (triggered by Unit28) is presented in Figure 9D. In the control condition, when several EPSPs triggered by this unit were superimposed, only the first depolarizing event was reliably present (Fig. 9D, Control) and therefore was likely attributable to a monosynaptic EPSP. This hypothesis is supported by the observation that the delay between the extracellularly recorded sensory spike and the onset of this first EPSP was constant. The second wave in the averaged EPSP was caused by polysynaptic events, which are characterized by nonconstant delays (Fig. 9D). On the averaged traces (Fig. 9C, Unit28), the rapid likely monosynaptic EPSP was increased from $0.832 \pm 0.022 \mathrm{mV}(n=70)$ in control to $0.991 \pm 0.051 \mathrm{mV}(n=$ $62)$ in the $5 \mathrm{HT}$ condition. This increase was significant $(t$ test; $p=$ 0.039 ) (Table 1). In addition, the amplitude of the late compound EPSP (measured $6 \mathrm{msec}$ after the peak of the monosynaptic EPSP) (Fig. 9C, arrow) was significantly increased from $0.428 \pm$ $0.075 \mathrm{mV}$ in control to $0.980 \pm 0.130 \mathrm{mV}$ in the $5 \mathrm{HT}$ condition $(t$ test; $p=0.0002$ ). This increase was a result of the recruitment of large and late polysynaptic EPSPs after 5HT application (Fig. 9D, $5 \mathrm{HT}$ ). Similar results were obtained in 12 preparations (Table 2).

To verify that EPSP amplitude did not depend on the phase of 


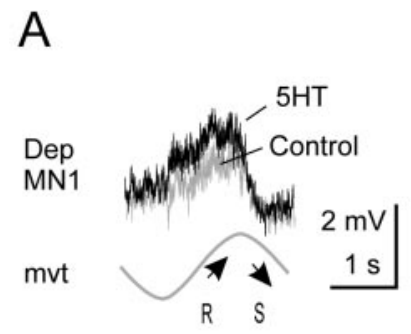

C
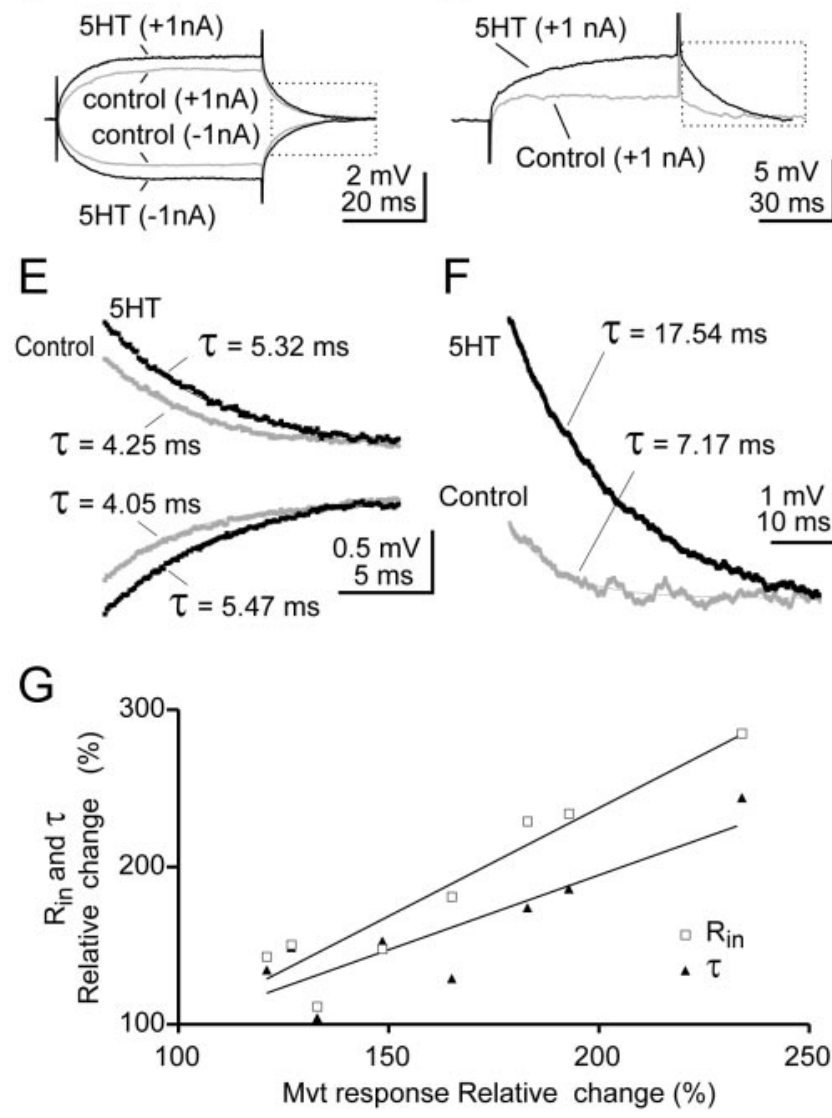

Figure 8. $5 \mathrm{HT}$ increases input resistance in Dep MNs. $A, B$, In two intracellularly recorded Dep MNs ( $A$, Dep MN1; $B$, Dep MN2), sinewave movements (mvt) applied to CBCO strand evoked a typical resistance reflex response (gray trace, Control) that was increased in the presence of $5 \mathrm{HT}$ (black trace, 5HT). Each trace is an average of $n=12$ cycles. $C, D$, Measurement of the input resistance of the two Dep MNs recorded in $A$ and $B$. In both cases, the input resistance increased in the presence of 5 HT. E, F, Repolarization time constant $(\tau)$ measured from (and $D$ (see boxes). Estimations of $\tau$ were made on average traces $(n=10)$ after fitting each recording with one exponential decay curve $\left(R^{2}>0.95\right)$. The first few milliseconds were not taken into account for the calculation. Experiments in which the electrode capacitance was too high were rejected. In both cases, time constant was increased in the presence of 5HT. G, Relative changes in reflex response (percentage of control response) are correlated with changes in input resistance $\left(R_{i n}\right.$ open squares; $R^{2}=0.91$ ) and changes in time constant ( $\tau$, filled triangles; $R^{2}=0.76$ ). Moreover, $R_{\text {in }}$ and $\tau$ were significantly paired (paired $t$ test; $p<0.05$ ).

the sinewave movement imposed on the CBCO strand, which could mask some of the effects of 5HT on unitary EPSPs, in some experiments we have systematically averaged the amplitude of unitary EPSPs with respect to the phase of the imposed movement. The result of such an analysis is shown in Figure 10. In the example presented, the global resistance reflex response of the Dep MN was increased by $94.8 \%$ in the $5 \mathrm{HT}$ condition. One unitary EPSP is presented (Fig. 10A). The amplitudes of the averaged EPSPs of this unit in control $(0.590 \pm 0.016 \mathrm{mV} ; n=526)$

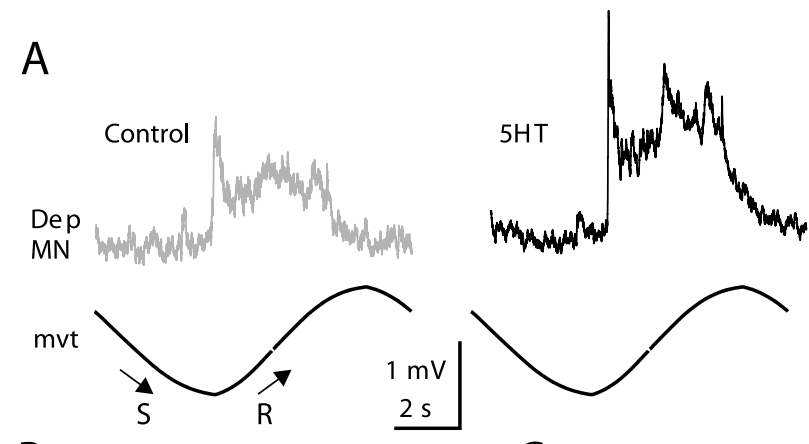

B
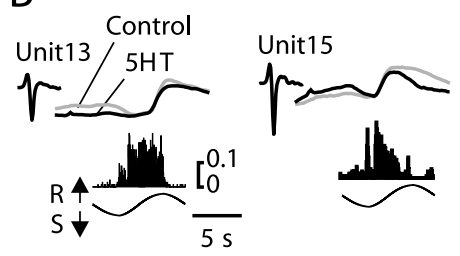

C
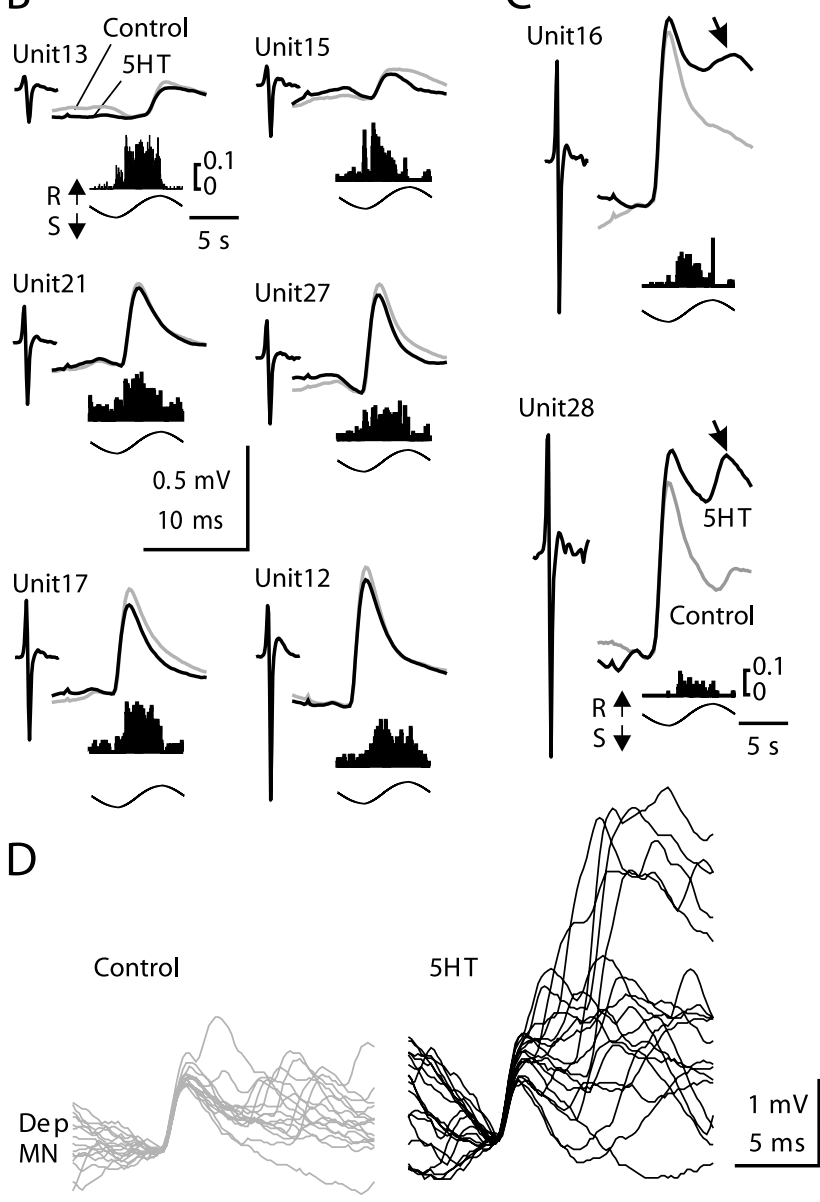

Figure 9. Effect of 5HT on unitary EPSPs recorded from a Dep MN and triggered by releasesensitive $C B C O$ units. $A$, Mean response of a Dep MN to 10 sinewave movements applied to the $\mathrm{CBCO}$ strand in control and $5 \mathrm{HT}$ conditions. $B, C$, Average traces of unitary EPSPs recorded from the Dep MN shown in $A$ (for statistics, see Table 1). Each unitary EPSP was triggered by a CBCO unit (Unit) extracellularly recorded from the $\mathrm{CBCO}$ nerve. The shape of each of these sensory units is presented on the left of each EPSP trace. The activity of each sensory unit during sinewave movement is represented by an event distribution histogram calculated over 10 sinusoidal movement cycles. Vertical scale represents the number of spikes per bin averaged per cycle (bin size, 50 msec; number of bins, 200). The movement cycle is represented below each graph. All of the units are activated during the release movement. For each unitary EPSP, two superimposed traces are presented corresponding to control (gray trace) and 5HT (black trace) conditions. Six EPSPs did not present any enhancement after $5 \mathrm{HT}$ application $(B)$, and two EPSPS displayed a marked enhancement (C).D, Superimposed Dep MN EPSPs $(n=18)$ triggered by Unit28 are presented in control (left) and $5 \mathrm{HT}$ (right) conditions.

and $5 \mathrm{HT}(0.580 \pm 0.017 \mathrm{mV} ; n=489)$ conditions were not significantly different $(t$ test; $p=0.663$ ) (Fig. $10 A$ ). Moreover, the plot of unitary EPSP amplitude against phase of the movement cycle demonstrates stationarity of EPSPs along movement cycle 
Table 1. Statistical analysis of amplitude of unitary EPSPs presented in Figure 9

\begin{tabular}{|c|c|c|c|}
\hline $\mathrm{CBCO}$ unit & $\begin{array}{l}\text { Control unitary EPSP mean } \\
\text { amplitude } \pm \text { SEM }\end{array}$ & $\begin{array}{l}\text { 5HT unitary EPSP mean am- } \\
\text { plitude } \pm \text { SEM }\end{array}$ & $t$ test $p$ value \\
\hline & & & $p<$ \\
\hline Unit12 & $\begin{array}{l}0.657 \pm 0.0013 \\
n=567\end{array}$ & $\begin{array}{l}0.564 \pm 0.019 \\
n=483\end{array}$ & $0.0001^{* * * *}$ \\
\hline Unit13 & $\begin{array}{l}0.153 \pm 0.015 \\
n=737\end{array}$ & $\begin{array}{l}0.128 \pm 0.015 \\
n=856\end{array}$ & $\begin{array}{l}p=0.244 \\
\text { NS }\end{array}$ \\
\hline Unit15 & $\begin{array}{l}0.137 \pm 0.016 \\
n=436\end{array}$ & $\begin{array}{l}0.115 \pm 0.017 \\
n=424\end{array}$ & $\begin{array}{l}p=0.335 \\
\text { NS }\end{array}$ \\
\hline Unit16 & $\begin{array}{l}0.759 \pm 0.020 \\
n=240\end{array}$ & $\begin{array}{l}0.788 \pm 0.021 \\
n=273\end{array}$ & $\begin{array}{l}p=0.332 \\
\text { NS }\end{array}$ \\
\hline Unit17 & $\begin{array}{l}0.478 \pm 0.010 \\
n=575\end{array}$ & $\begin{array}{l}0.419 \pm 0.014 \\
n=606\end{array}$ & $p=0.0008^{* * *}$ \\
\hline Unit21 & $\begin{array}{l}0.368 \pm 0.012 \\
n=737\end{array}$ & $\begin{array}{l}0.348 \pm 0.014 \\
n=677\end{array}$ & $\begin{array}{l}p=0.301 \\
\text { NS }\end{array}$ \\
\hline Unit27 & $\begin{array}{l}0.496 \pm 0.012 \\
n=607\end{array}$ & $\begin{array}{l}0.453 \pm 0.015 \\
n=607\end{array}$ & $p=0.026^{*}$ \\
\hline Unit28 & $\begin{array}{l}0.832 \pm 0.022 \\
n=70\end{array}$ & $\begin{array}{l}0.991 \pm 0.051 \\
n=62\end{array}$ & $p=0.0039^{* *}$ \\
\hline
\end{tabular}

(Fig. $10 B, C$ ). The cycle was divided into 10 bins. The mean value and the variance of the amplitude per bin did not significantly change along the cycle (Fig. 10C). Note that in such analysis, the bins with too few events were not taken into account (Fig. 10C, open bars). This estimation was based on the analysis of the frequency distribution of the events in the movement cycle (Fig. $10 \mathrm{D})$. Moreover, the two classes of EPSPs occurred during opposite movements of the CBCO: the EPSPs counted in filled bars occurred during the release movement of the CBCO (resistance reflex EPSPs), whereas the EPSPs counted in open bars occurred during the opposite movement (stretch of the CBCO-downward movement of the leg in the intact animal) and therefore occurred in conjunction with IPSPs of the inhibitory component of the resistance reflex (Le Bon-Jego and Cattaert, 2002). Their amplitude therefore was generally smaller, and they were not included in the analysis. Similar results were obtained with all of the identified unitary EPSPs analyzed $(n=37)$.

\section{The increase of resistance reflex response involves polysynaptic pathways}

To estimate the role of polysynaptic pathways in the 5HTinduced increase of the resistance reflex response, we performed a series of experiments in which the CNS was bathed successively in normal saline and in a high divalent cation solution, both in control and in 5HT conditions. The response of an intracellularly recorded Dep $\mathrm{MN}$ to $\mathrm{CBCO}$-imposed sinewave movement is presented in Figure $11 A$. In high divalent cation solution (Fig. 11A2), the amplitude of the control response (Fig. $11 \mathrm{Al}$ ) was reduced from $0.96 \pm 0.04$ to $0.67 \pm 0.02 \mathrm{mV}$, indicating that even in the absence of exogenous 5HT, polysynaptic pathways are involved in the global resistance reflex response. When back in control saline (Fig. 11A3), the amplitude of the resistance reflex response was not totally restored to its initial value $(0.78 \pm$ $0.02 \mathrm{mV})$. Application of 5HT $(10 \mu \mathrm{M}$ in normal saline) induced a large increase of the amplitude of the resistance reflex response $(1.43 \pm 0.07 \mathrm{mV})$ (Fig. 11A4). This increase disappeared when the CNS was bathed with a high divalent cation solution $(0.78 \pm 0.02 \mathrm{mV})$ (Fig. 11 A5), indicating that the effect of $5 \mathrm{HT}$ was caused by a change in the polysynaptic pathways. Statistic analysis using one-way ANOVA and Tukey's multiple comparison test confirmed that the difference between control and 5HT conditions when the CNS was bathed in control saline (Fig. 11B1) was highly significant ( $p<0.001)$. By contrast, in high divalent conditions, $5 \mathrm{HT}$ induced no significant change in the reflex response ( $p>0.05)$ (Fig. 11B2).

During this series of experiments, an analysis of unitary EPSPs was made. Two examples of this analysis are given in Figure $11 C$. EPSPs obtained in control, 5HT, and 5HT in the presence of high divalent cation solution are superimposed for the two examples. These examples were chosen because they are representative of two categories of effects on unitary EPSPs. In both examples, the EPSPs presented a simple shape in control condition (i.e., a single fast depolarizing phase followed by a slow recovery phase). After 5HT application, the peak amplitude of the EPSP triggered by Unit1 increased from $0.22 \pm 0.02$ to $0.31 \pm 0.03 \mathrm{mV}$ (Fig. 11C1). This significant increase of the amplitude of the early EPSP was observed in $17.2 \%$ of the unitary EPSPs analyzed. The second category is represented by the EPSP triggered by Unit2 (Fig. $11 C 2)$, in which the peak amplitude was slightly reduced (0.82 \pm 0.11 to $0.72 \pm 0.12 \mathrm{mV}$ ) (Fig. 11C2). Such effects were observed in $23.9 \%$ of analyzed EPSPs. Although the general shape of these EPSPs did not change (there was no additional late polysynaptic EPSP recruited) after 5HT application (compare traces labeled "25 min 5HT" and "Control"), their recovery phase was significantly slower (in control situation, $\tau=3.12$ and $3.15 \mathrm{msec}$ for EPSPs triggered by Unit1 and Unit2, respectively; after 5HT application: $\tau=6.51$ and $6.49 \mathrm{msec}$ for units 1 and 2, respectively). When the CNS was bathed with high divalent cation solution, these changes were abolished (Fig. 11C, dashed lines), indicating that $5 \mathrm{HT}$ induced modifications of the recovering phase of EPSPs involved in polysynaptic pathways. Note that although the shape of the EPSP triggered by Unit1 remained simple after 5HT application, the peak amplitude increase was a result of the recruitment of polysynaptic events. Therefore, the "simple shape" feature was not an indication of a purely monosynaptic event, and subsequently we systematically tested for the presence of polysynaptic pathways in unitary EPSPs in several experiments. In all of these experiments $(n=22)$, each time an EPSP presented a no-

Table 2. Effect of 5 HT on the amplitude of unitary EPSPs in 12 experiments

\begin{tabular}{|c|c|c|c|c|c|c|c|}
\hline \multirow[b]{2}{*}{ Experiment } & \multirow{2}{*}{$\begin{array}{l}\text { Number of } \mathrm{CBCO} \text { units } \\
\text { evoking an EPSP in } \\
\text { the MN }\end{array}$} & \multicolumn{3}{|c|}{ Effect of $5 \mathrm{HT}$ on early EPSP } & \multicolumn{3}{|c|}{ Effect of $5 \mathrm{HT}$ on late EPSP ${ }^{a}$} \\
\hline & & No change & Increase & Decrease & No change & Increase & Decrease \\
\hline 1 & 8 & 4 & 1 & 3 & 0 & 2 & 0 \\
\hline 2 & 6 & 4 & 0 & 2 & 0 & 2 & 0 \\
\hline 3 & 8 & 6 & 1 & 1 & 0 & 3 & 0 \\
\hline 4 & 8 & 5 & 1 & 2 & 1 & 6 & 0 \\
\hline 5 & 8 & 3 & 0 & 5 & 0 & 4 & 0 \\
\hline 6 & 4 & 4 & 0 & 0 & 0 & 2 & 0 \\
\hline 7 & 6 & 5 & 1 & 0 & 0 & 2 & 0 \\
\hline 8 & 7 & 2 & 4 & 1 & 0 & 5 & 0 \\
\hline 9 & 11 & 7 & 3 & 1 & 0 & 4 & 0 \\
\hline 10 & 4 & 1 & 3 & 0 & 0 & 2 & 0 \\
\hline 11 & 4 & 4 & 0 & 0 & 1 & 1 & 0 \\
\hline 12 & 6 & 2 & 0 & 4 & 0 & 2 & 0 \\
\hline
\end{tabular}

For each experiment, the number of EPSPs presenting a type of response to $5 \mathrm{HT}$ (no effect, increase, decrease) is given. We have distinguished the early EPSPS from the late compound EPSPs. The significance of effects was assessed by $t$ test as in Table 1 , with $p<0.05$.

${ }^{a}$ Only a fraction of the analyzed EPSPs presented a clearly visible late compound EPSP. 
A

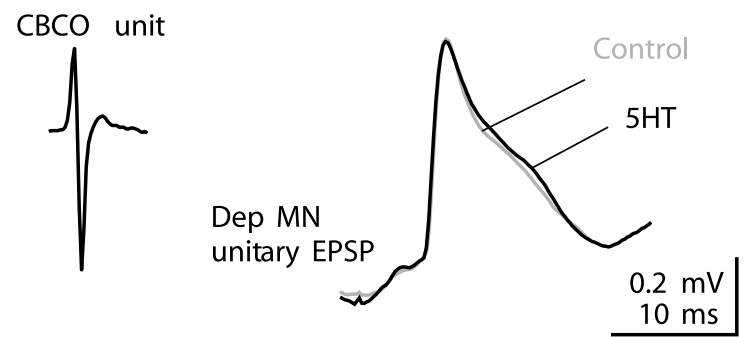

B

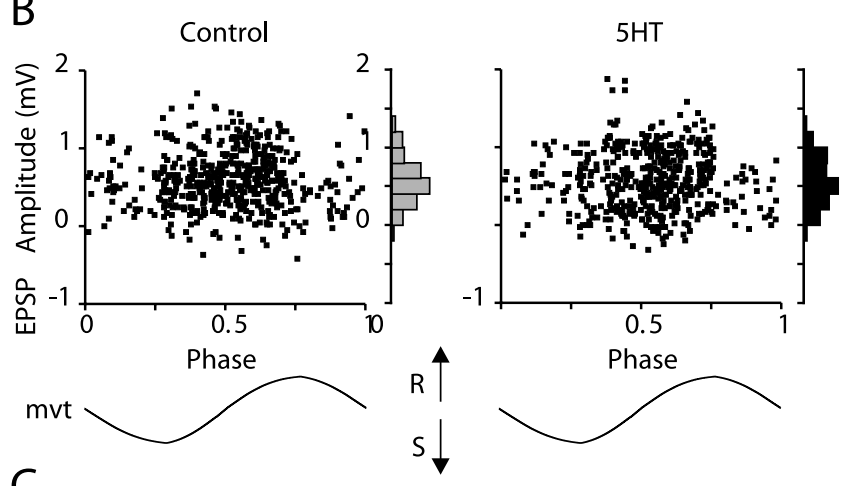

C
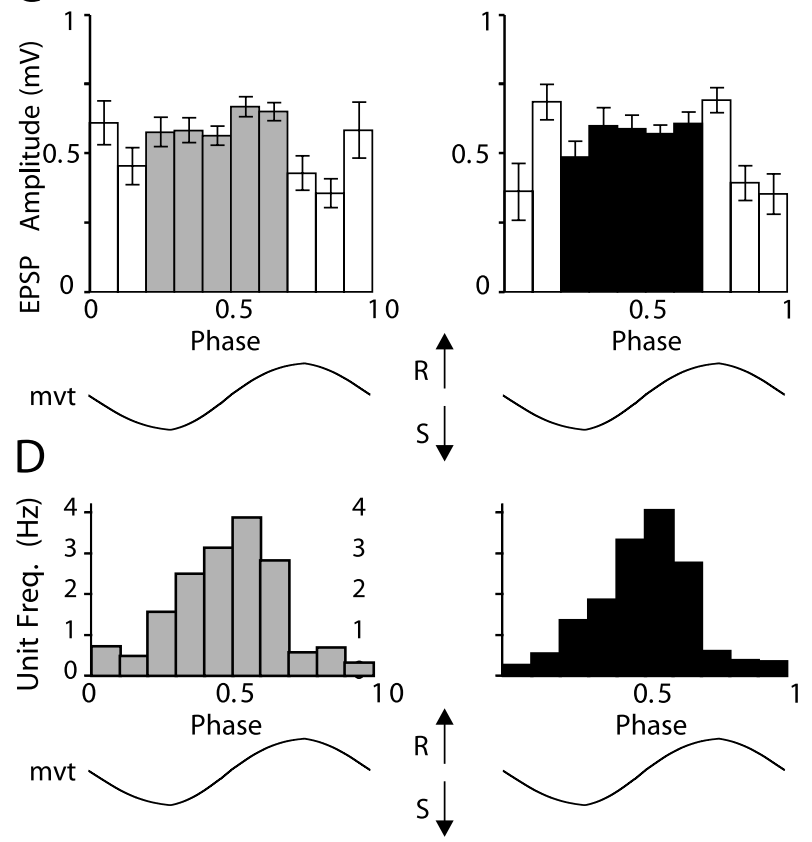

Figure 10. Stationarity of unitary EPSP amplitude during release movement. $A$, Averaged unitary Dep MN EPSP triggered by a $C B C 0$ unit extracellularly recorded from the $C B C 0$ nerve in control (gray line; $n=735$ ) and 5HT (black line; $n=675$ ) conditions. $B, C$, Statistical analysis of the amplitude of this unitary EPSP against the phase of imposed movement. B, Plot of the amplitude of all unitary EPSPs recorded during 300 sec in control (left) and $5 \mathrm{HT}$ (right) conditions, against the phase of the sinewave movement imposed on the $\mathrm{CBC} O$ strand (mvt). Distribution histograms are presented on the right of each plot. C, Histograms representing the mean amplitude of unitary EPSPs against the phase of imposed movement. The movement cycle was divided into 10 bins. Error bars represent SE. Open bars represent discharge frequency of the unit below $1 \mathrm{~Hz}$. D, Frequency distribution of the events (Unit Freq.) along the phase of imposed movement.

ticeable increase of peak amplitude or recovery time constant, this was a result of polysynaptic events as in Figure $11 C$.

\section{HT and EPSP amplitude}

In the presence of 5HT the input resistance of the MNs was generally increased by a factor 1.2-2.9 (Fig. 8G). However, during the

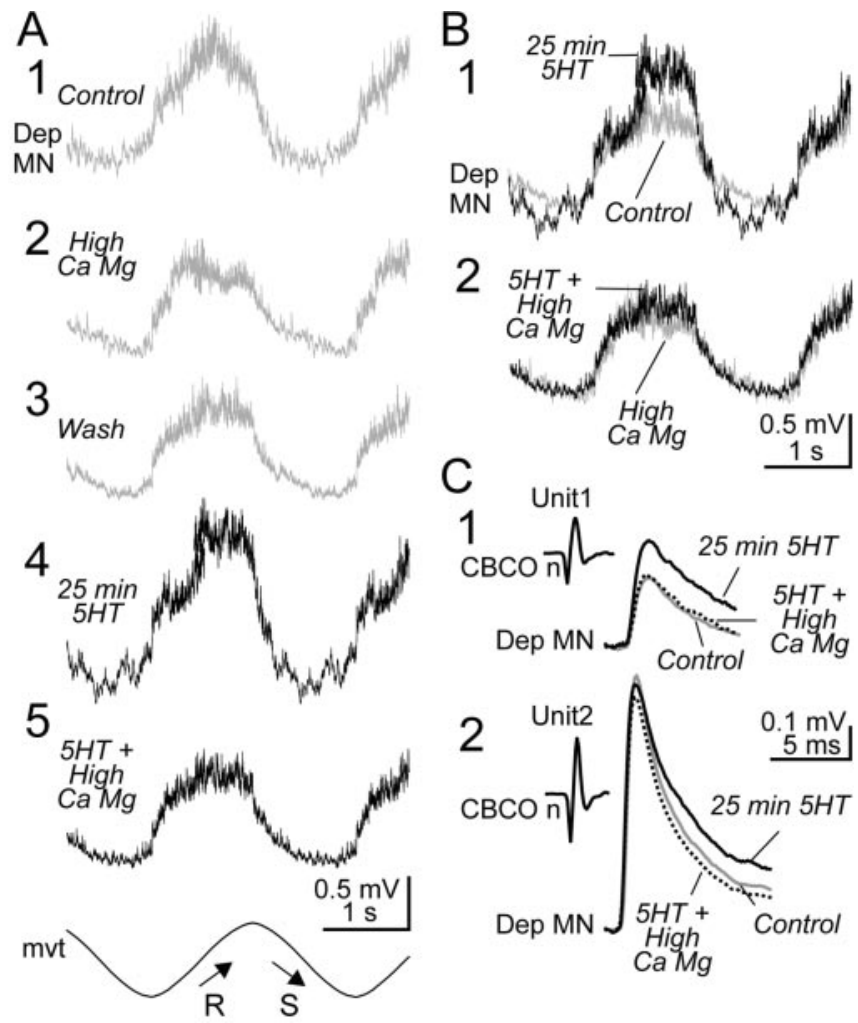

Figure 11. Polysynaptic pathways are involved in the 5HT-induced enhancement of the resistance reflex response. $A$, Intracellular averaged $(n=13)$ responses of a Dep MN to application of a sinewave movement to the $\mathrm{CBCO}$ strand in successive conditions. $A 1$, The intracellularly recorded Dep MN displays a resistance reflex response (control). $A 2$, In the presence of the high divalent cation solution, the amplitude of the control response is decreased (High Ca Mg). A3, After 40 min washing (Wash) of the high divalent cation solution, the Dep MN response is not restored. $A 4$, When $5 \mathrm{HT}$ is applied, the amplitude of the response is greatly increased (25 $\min 5 \mathrm{HT})$. A5, This large increase disappears in the presence of high divalent cation solution $(5 \mathrm{HT}+\mathrm{High} \mathrm{Ca} \mathrm{Mg}) . \mathrm{B}$, Comparison of the effects of $5 \mathrm{HT}$ in normal saline and in a high divalent cation solution on the Dep MN recorded in $A . B 1$, In normal saline, $5 \mathrm{HT}$ induces an increase in the resistance reflex response. $B 2$, In a high divalent cation solution, $5 \mathrm{HT}$ has almost no effect on the resistance reflex response. $C$, Averaged unitary EPSPs triggered by two $\mathrm{CBCO}$ units ( $C 1$ and $C 2$; same procedure as in Fig. $9 B, C$ and recorded from the same Dep MN as in $A$ and $B$. These two unitary EPSPs were recorded in three conditions, and the averaged traces $(n=50)$ corresponding to control (Control, gray trace), $5 \mathrm{HT}$ ( $25 \mathrm{~min} 5 \mathrm{HT}$, black trace), and $5 \mathrm{HT}$ in the presence of high divalent cation solution (5HT + High Ca Mg, dotted line) are superimposed.

application of movements to the CBCO strand, many EPSPs did not increase their amplitude in the presence of 5HT (Fig. 9, Table 1). This intriguing result could be caused by three phenomena. (1) in the presence of $5 \mathrm{HT}$, the membrane potential of the $\mathrm{MN}$ is more depolarized than in the control condition (Figs. 3B, 4, 6). Therefore, the electrotonic driving force supporting the EPSPs is smaller in 5HT condition, and the amplitude of EPSPs is expected to become smaller. (2) Because of massive synaptic inputs, the input resistance may be locally reduced (shunting excitation), causing the decrease of EPSP amplitude. (3) During a volley of EPSPs, each EPSP summates with the falling phase of preceding EPSPs, resulting in a smaller EPSP amplitude as frequency increases. We have analyzed these hypotheses. A simulation study demonstrated that the small change in driving force caused by the slightly more depolarized membrane potential in the 5HT condition did not contribute significantly to the change in EPSP amplitude observed (data not shown). Similarly, the effect of shunting excitation was tested in simulation studies, and it was concluded that it did not contribute significantly to the change in 
A

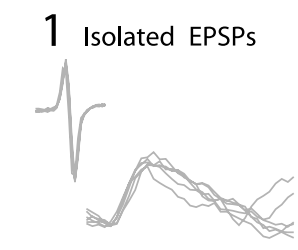

B

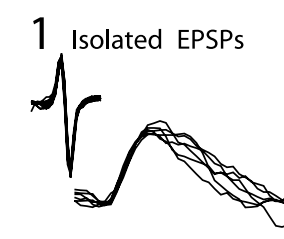

C

1 Isolated EPSPS

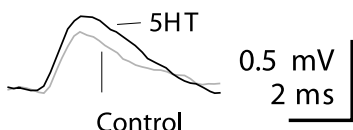

Control

$5 \mathrm{HT}$
Control
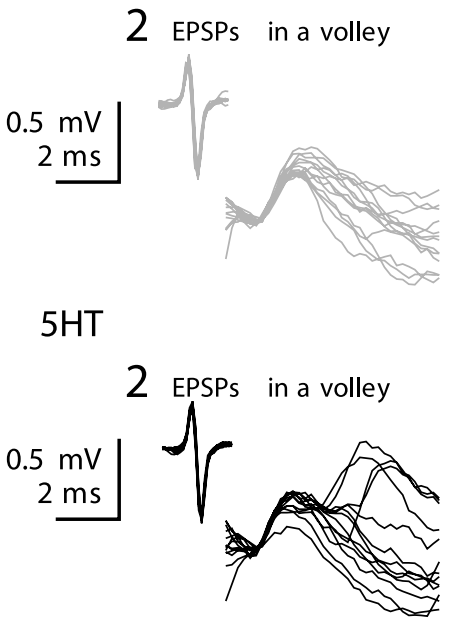

2 EPSPs in a volley

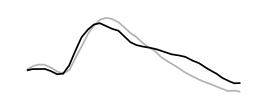

Figure 12. Effect of 5HT on isolated EPSPs. A-C, Intracellular recordings from a Dep MN showing unitary EPSPs triggered by a $\mathrm{CBCO}$ unit in control condition ( $A$, gray traces) and after $5 H T$ application (B, black traces). In $A 1$ and $B 1$ are presented six superimposed EPSPs (raw data) recorded in the absence of movement (isolated EPSPs). In $A 2$ and $B 2$ are presented 13 superimposed EPSPs (raw data) recorded during sinewave movements applied to the CBCO strand (EPSP in a volley). C, Average traces ( $n=150$ for each trace) of the unitary EPSPs shown in $A$ and $B$ (gray traces, control condition; black traces, after $5 \mathrm{HT}$ application).

EPSP amplitude observed (data not shown). The decrease in EPSP amplitude was caused mainly by an increase of EPSP frequency in the volley in the presence of 5HT. Indeed, during a volley, the amplitude of each EPSP summates with the falling phases of preceding EPSPs. This phenomenon is illustrated in Figure $13 A$, in which the amplitude $\left(V_{1}\right)$ of the second EPSP is smaller than the amplitude $\left(V_{0}\right)$ of the first EPSP, because the second EPSP summates with the falling phase of the first EPSP. The amplitude of subsequent EPSPs is decreased further because of accumulation of falling phases (Fig. 13B). Because of this phenomenon, increasing the frequency of the volley results in a further decrease in the amplitude of EPSPs.

This hypothesis was tested in EPSPs intracellularly recorded from Dep MNs. When amplitude measurements were performed on an isolated identified unitary EPSP in the absence of movement stimulation (in this condition most of the sensory neurons were silent or, when active, their discharge frequency was less than a few hertz), the amplitude of such an "isolated" EPSP was always increased in preparation in response to $5 \mathrm{HT}$ application (Fig. 12). In the experiment reported here. EPSPs from a sensory neuron have been recorded in four conditions: in control situation and in the presence of 5HT both during and in the absence of sinewave movement imposed on the CBCO. In the control situation, in the absence of movement, this sensory neuron produced a few sensory spikes (mean frequency $=2 \mathrm{~Hz}$ calculated over a period of $15 \mathrm{~min}$ ). These sensory spikes produced EPSPs (Fig. $12 \mathrm{~A} 1$, Isolated EPSPs). During a series of sinewave movements imposed on the CBCO, this same sensory neuron presented a burst of sensory spikes that evoked a volley of EPSPs in the Dep

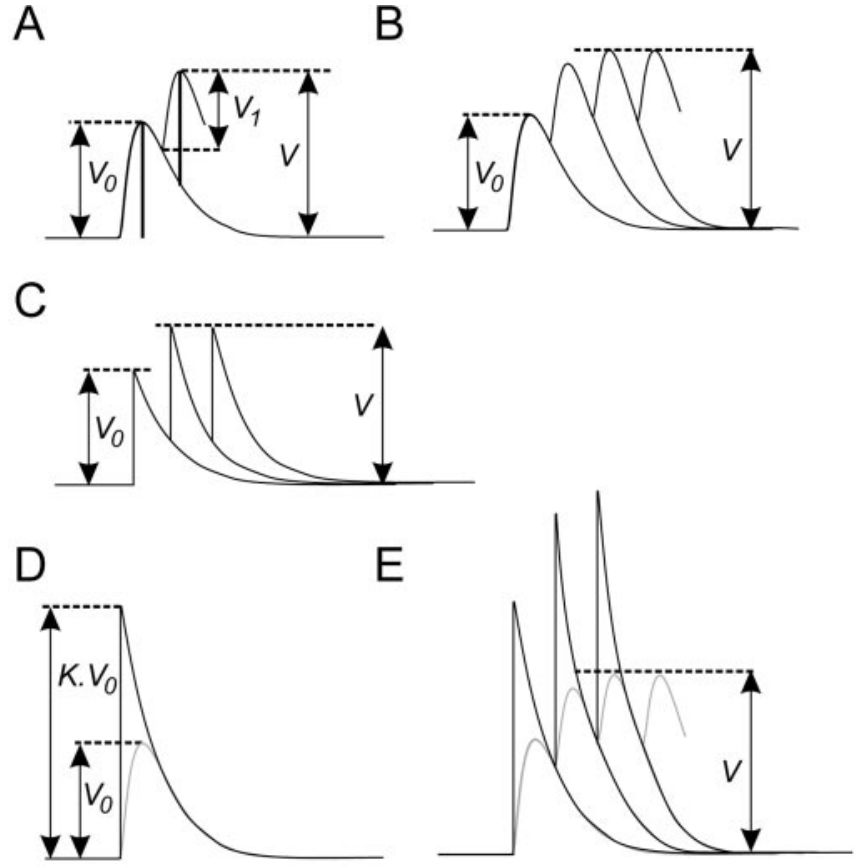

Figure 13. Model of temporal summation. $A$, In a volley containing only two EPSPs, the amplitude $\left(V_{1}\right)$ of the second EPSP is smaller than the amplitude $\left(V_{0}\right)$ of the first EPSP, because the second EPSP summates with the falling phase of the first EPSP. $B$, In a volley of $n$ EPSPs, the maximum amplitude $(V)$ of the volley reaches rapidly a steady state. In this steady state, the amplitude of each EPSP is smaller than the amplitude $V_{0}$ of the first EPSP or an isolated EPSP.C, Modeling of EPSPs by an instantaneous depolarization $\left(V_{0}\right)$ followed immediately by a single exponential falling phase (see Results for explanations). D, E, More realistic model of the EPSP summation. $D$, To model the delay attributable to the discrete rising phase, the amplitude of the model EPSP was artificially increased by a factor $K$. With this new amplitude $\left(K . V_{0}\right)$, the falling phase of the realistic model EPSP (black trace) fits perfectly the real EPSP falling phase (gray trace). $E$, With this more realistic model, the summation of EPSPs (black traces) fits the summation of real shape EPSPs (gray traces). The maximum amplitude $(V)$ is obtained by the addition of an EPSP with an amplitude $V_{0}$ to the series of model EPSPs with an amplitude $K . V_{0}$.

MN (data not shown). These volley EPSPs are shown in Figure $12 \mathrm{~A} 2$. Isolated EPSPs were significantly larger ( $t$ test; $p<0.0001)$ in the $5 \mathrm{HT}$ condition $(0.458 \pm 0.013 \mathrm{mV})$ than in control $(0.372 \pm$ $0.011 \mathrm{mV}$ ) (Fig. 12 B1,C1), and the likely monosynaptic part of the volley EPSPs was significantly smaller $(t$ test; $p=0.0176)$ in the $5 \mathrm{HT}$ condition $(0.311 \pm 0.016 \mathrm{mV})$ than in control $(0.365 \pm 0.015 \mathrm{mV})$ (Fig. 12 B2,C2). Such measurements were not possible on most of the $\mathrm{CBCO}$ units because they were activated only during movement application. Only 12 units projecting on Dep MNs and presenting a slow rate of tonic activity in the absence of sinewave movement were analyzed. For all of these units, isolated EPSPs were significantly larger in the 5HT condition than in the control condition. For six of these units the volley EPSP was significantly smaller in 5HT condition than in control. For four of these units there was no significant difference in volley EPSP between control and 5HT conditions. Two units displayed larger volley EPSPs in 5HT condition.

\section{Integrative properties of MNs}

The integrative properties of a neuron and its input-output relationship depend on several parameters such as input resistance, EPSP frequency, time constant, and membrane potential. In all of the experiments in which $5 \mathrm{HT}$ induced an increase in the resistance reflex, an increase in the input resistance of the Dep MN was observed (Fig. 8). To understand the effect of this parameter on the integrative properties of the $\mathrm{MN}$, we will examine first the relationship between the shape of an EPSP and the summation 
properties of the MN. Let us consider a volley of $n$ EPSPs at a frequency $N$. Each EPSP has a decay time constant $\tau$. For simplicity, suppose that each EPSP can be modeled as an instantaneous rising phase followed by an exponential decay. The last EPSP will summate with the exponentially decreasing trace of the previous EPSP. In the case of a volley containing only two EPSPs (Fig. $13 A$ ), the maximum amplitude of depolarization $(V)$ reached during the volley by the second EPSP is:

$$
V=V_{0}+V_{0} \times e^{\frac{-t}{\tau}}
$$

with

$$
t=\frac{1}{N}
$$

where $V_{0}$ is the amplitude of a single EPSP.

In a volley containing n EPSPs (Fig. 13B), we summate all preceding EPSPs:

$$
\begin{gathered}
V=V_{0}+V_{0} \times e^{\frac{-1}{N \tau}}+V_{0} \times e^{2 \times \frac{-1}{N \tau}}+ \\
V_{0} \times e^{3 \times \frac{-1}{N \tau}}+\cdots+V_{0} \times e^{n \times \frac{-1}{N \tau}} \\
V=V_{0} \times\left(1+e^{\frac{-1}{N \tau}}+e^{2 \times \frac{-1}{N \tau}}+e^{3 \times \frac{-1}{N \tau}}+\cdots+e^{n \times \frac{-1}{N \tau}}\right) .
\end{gathered}
$$

In an infinite series of EPSPs:

$$
\begin{gathered}
V=V_{0} \times\left(1+X+X^{2}+X^{3}+\cdots\right. \\
\left.+X^{\infty}\right)=V_{0} \times\left(\frac{1}{1-X}\right), X=e^{\frac{-1}{N \tau}} \\
V=V_{0} \times\left(\frac{1}{1-e^{\frac{-1}{N \tau}}}\right) .
\end{gathered}
$$

The value of $V$ depends on the value of $e^{\frac{-1}{N \tau}}$. If this value is close to $1, V$ will be very large, but generally this value is small because of the small time constant $\tau$. In most of our recordings $\tau$ was $<10$ msec $\left(10^{-2} \mathrm{sec}\right)$. Therefore, with a frequency of $100 \mathrm{~Hz}$ (which is on the order of magnitude of EPSP frequency received by a given Dep MN), $N \tau=1$, and $\frac{-1}{e^{N \tau}}$. $=0.37$, then $V=V_{0} \times 1.58$.

This model is very simple and underestimates temporal summation because we considered each EPSP to have an instantaneous rising phase followed by a simple exponential decay that begins immediately (Fig. 13C). In fact, a real EPSP has a discrete rising phase that causes a delay in the onset of the exponential falling phase. To model this delay using EPSPs with single exponential falling phases, we artificially increased by a factor $K$ the amplitude of all EPSPs preceding the final EPSP that was used in the measurement of $V$ (Fig. $13 D, E$ ); then the estimation of $V$ can be written for $n$ EPSPs as:

$$
\begin{aligned}
V=V_{0}+K \times V_{0} \times e^{\frac{-1}{N \tau}}+ & K \times V_{0} \times e^{2 \times \frac{-1}{N \tau}}+K \times V_{0} \\
& \times e^{3 \times \frac{-1}{N \tau}}+\cdots+K \times V_{0} \times e^{n \times \frac{-1}{N \tau}},
\end{aligned}
$$

and with an infinite number of EPSPs:

$$
\begin{aligned}
V=V_{0}+K \times V_{0} \times X\left(1+X+X^{2}+\right. & \left.X^{3}+\cdots+X^{\infty}\right)=V_{0} \\
& +K \times V_{0} \times\left(\frac{X}{1-X}\right) .
\end{aligned}
$$

In this new expression, with the same parameters $(\tau=10 \mathrm{msec}$; $n=100 \mathrm{~Hz}$ ), we get a depolarization $V=V_{0} * 2.16$ during the 100 $\mathrm{Hz}$ volley. The maximum amplitude is directly proportional to the amplitude of EPSPs, whereas the two other parameters ( $\tau$ and $n$ ) are equally important and induce a nonlinear effect on summation.

Let us now examine the effect of 5HT on these parameters. We can hypothesize that any change in input resistance of the MN will result in a change of the same order of magnitude in the amplitude of EPSPs. If this is true, then in the presence of 5HT, the amplitude of EPSP should be increased by a factor of 1.2-2.9 (Fig. 8G). This first effect on the amplitude of EPSPs will directly produce an increase in the global response to movement of the same order of magnitude. In addition, 5HT will also increase the decay time constant $\tau$ of EPSPs, which will increase the global response to movement during the volley of EPSPs. Finally, in the 5HT condition, polysynaptic EPSPs are added to the monosynaptic EPSPs. This addition is equivalent to increasing the frequency $n$. For example, if the decay time constant $\tau$ of EPSPs is doubled (from $\tau=7 \mathrm{msec}$ to $14 \mathrm{msec}$, with $n=100 \mathrm{~Hz}$ ) then $X=e^{\frac{-1}{N \tau}}$ is increased from 0.24 to 0.49 , and the global response is increase from 0.81 to $1.46 \mathrm{mV}$ ( $80 \%$ increase). If we add now the polysynaptic EPSPs $(n=200 \mathrm{~Hz}), X$ increases to 0.70 and the global response increases to $2.83 \mathrm{mV}$ ( $247 \%$ increase). It is notable that these values are compatible with the experimental observations of the global response in the presence of 5HT (Fig. 8G). Thus realistic changes in the parameters $(n$ and $\tau$ ) can result in considerable increase in summation.

On the other hand, the magnitude of the effects of increasing $n$ or $\tau$ will depend on the values of these parameters. If $n$ is too small, summation will not be very effective because of the exponential form of $X\left(X=e^{\frac{-1}{N \tau}}\right)$, and in this case, increasing $\tau$ may not lead to a noticeable increase in the global response. For example, when the polysynaptic excitatory pathways are blocked, the frequency of synaptic input $(n)$ is lower, leading to less temporal summation. This phenomenon was likely responsible for the small, statistically insignificant increase in the global response to $5 \mathrm{HT}$ in high divalent cation solution shown in Figure $11 \mathrm{B2}$ ( $0.67 \pm 0.02 \mathrm{mV}$ in control to $0.78 \pm 0.02 \mathrm{mV}$ in $5 \mathrm{HT}$ condition), although $\tau$ was increased by $5 \mathrm{HT}$.

\section{Discussion}

We demonstrated that in the CNS 5HT modulates proprioceptive sensorimotor integration in crayfish walking legs. Indeed, 5HT enhances the resistance reflex involved in posture control via two main effects: (1) increasing input resistance of the Dep MN and (2) activation of the polysynaptic pathway (Figs. 9D, 11).

\section{Effect of 5HT on MNs}

The two effects of 5HT on Dep MNs (slow tonic depolarization and increase in input resistance) occur with different time scales. Depolarization started after $1 \mathrm{~min}$ in the presence of $5 \mathrm{HT}$ and reached a maximum after $5 \mathrm{~min}$. In a series of experiments in which $5 \mathrm{HT}$ was pressure applied close to an intracellularly recorded Dep MN, each puff of 5HT elicited a rapid (a few tens of milliseconds) and transient depolarization (D. Cattaert, unpublished observations). Such 5HT-induced depolarization was observed in other preparations (Katz and Harris-Warrick, 1989; McCormick and Pape, 1990; Kiehn and Harris-Warrick, 1992). The second effect of 5HT on Dep MNs 
consisted of an increase in the input resistance. In our experiments, this effect was detectable after $10 \mathrm{~min}$ in the presence of $5 \mathrm{HT}$ and reached a maximum after $25 \mathrm{~min}$. Because of the long latency of this effect, the input resistance increase is likely mediated through metabotropic 5HT receptors and second messengers. The multiplicity of effects of 5HT on a given target was shown in the stomatogastric ganglion of the crab, in which 5HT evoked a depolarization in dorsal gastric (DG) neurons that was accompanied by a decrease in input conductance (Kiehn and Harris-Warrick, 1992). The authors demonstrated that 5HT modulated more than one target conductance in DG neurons and that the effect depended on the membrane potential of DG. In addition, in the escape circuit of the crayfish, $5 \mathrm{HT}$ induces dual opposite effects depending on the mode of application (Teshiba et al., 2001). In the present work, 5HT was applied with a slow perfusion system, and the level of 5HT increased gradually to reach $10 \mu \mathrm{M}$ in the bath. Therefore, our findings are in accordance with the effects of low doses of $5 \mathrm{HT}$ on the escape circuit of the crayfish.

\section{Effect of 5HT on the polysynaptic excitatory pathway}

The polysynaptic pathways activated in the presence of 5HT involved spiking interneurons because they were blocked in the presence of high divalent cation solution. This result indicates that in the presence of 5HT, the involved spiking interneurons increased their excitability, allowing their membrane potential to reach the threshold for spike generation, and thereby evoked an EPSP in postsynaptic MNs. Such a 5HT-induced increase in excitability was demonstrated in rat facial MNs (Rasmussen and Aghajanian, 1990), pyramidal neurons from rat piriform cortex (Sheldon and Aghajanian, 1991), and neurons of the lamprey spinal cord (Van Dongen et al., 1986).

On the other hand, the increase in the polysynaptic response recorded from the MNs (Fig. 9) may result from an increase in the amplitude of the polysynaptic EPSPs. This later effect may result from the postsynaptic effect of 5HT on input resistance as analyzed in the mathematical model presented in this paper. However, the increase of EPSP amplitude in Dep MNs could also involve presynaptic mechanisms as was shown in rat dorsolateral septal nucleus via 5HT2A receptors (Hasuo et al., 2002).

\section{Multiplicity of 5HT effects}

It is notable that each of the 5HT-induced changes reported here would lead to minor effects if occurring alone, but that it is their conjunction that produces a dramatic enhancement of the resistance reflex. The small but consistent depolarization of the MNs will bring their membrane potential closer to spike threshold, thereby causing an increase in firing rate. The increase in the time constant of the MNs increases temporal summation of EPSPs and thereby allows the MNs to reach more depolarized potentials during a volley of EPSPs. The likely increase in excitability of the interneurons causes these neurons to fire more readily in response to a given sensory input and thereby reinforces the polysynaptic component of the compound EPSPs triggered by sensory spikes.

In addition to the central effects analyzed here, 5HT also induces peripheral effects on the sensory neurons and on muscles. It is striking that these peripheral effects act in the same direction as the central effects demonstrated here. When 5HT is bath applied on the CBCO proprioceptor, the excitability of the sensory neurons is increased (Rossi-Durand, 1993); however, the maxi- mal effect was obtained with a concentration of $1 \mu \mathrm{M}$. Higher concentrations seemed to be less effective, and for $20 \%$ of the recorded cells, $100 \mu \mathrm{M} 5 \mathrm{HT}$ induced a decrease of the sensory discharge. The duality of the effects on sensory neurons for 5HT concentrations of $10 \mu \mathrm{M}$ are in accordance with the present report on MNs. The significance of this dual effect is discussed below.

The other peripheral target of 5HT is the neuromuscular transmission. In the crayfish opener muscle, EPSP amplitudes increase up to 18-fold in the presence of 5-HT (Fischer and Florey, 1983). This effect was caused predominantly by a serotonininduced increase in the release of quanta of excitatory transmitter with nerve stimulation (Glusman and Kravitz, 1982). This increased synaptic transmission at the neuromuscular junction is use dependent (Qian and Delaney, 1997). In the locust, 5HT was also shown to increase muscle contraction. For example, bath application of $5 \mathrm{HT}$ on the mandibular closer muscles of the locust while the muscles are active produces marked changes in the contractions, increasing their amplitude, rate of contraction, and rate of relaxation (Baines et al., 1990). These authors have shown that $5 \mathrm{HT}$ elevates the levels of the cAMP in isolated muscle fibers.

All of these effects of $5 \mathrm{HT}$ seem to contribute to the same goal: increasing the resistance reflex activity. In the presence of 5HT, sensory neurons increase their sensitivity to movement, providing the CNS target neurons with higher rates of spike discharge. In the presence of 5HT, interneurons of polysynaptic pathways of the resistance reflex are more excitable and elicit large polysynaptic EPSPs in the MNs. In the presence of $5 \mathrm{HT}$, these MNs increase their responsiveness in at least two ways: a moderate tonic depolarization and an increased input resistance. As a consequence, their input-output relationship is steeper, and because of the increased sensory inflow relayed and amplified by interneurons of polysynaptic pathways, they are recruited more readily in response to any movement of the leg. As a consequence, previously silent MNs are activated. Finally, the two last stages (neuromuscular synaptic transmission and muscle contraction) are also enhanced in the presence of 5HT (Fischer and Florey, 1983; Baines et al., 1990). All of these combined effects may be caused by the activity of the 5 HT neurons that exist by pairs in thoracic and abdominal ganglia (Beltz and Kravitz, 1983). It is not known, however, whether these $5 \mathrm{HT}$-containing neurons project on the various targets mentioned here, and the modality of their discharge in the living animal is not known.

\section{Variability of 5HT-induced responses}

In experiments using simultaneous intracellular recordings from Dep MNs involved in resistance reflex, either all recorded Dep MNs displayed an increase of the resistance reflex or none of them was affected. Therefore, one source of variability of the response to 5HT is inter-individual. On the other hand, in a given experiment, the magnitude of the effect of 5HT was different among the different intracellularly recorded Dep MNs (Fig. 5), suggesting a variability between $\mathrm{MNs}$. This inter-MN variability could have several nonexclusive origins. First, the increase in the resistance reflex response can depend on the distance between the recording site and synapses of the polysynaptic pathway. Dep MNs indeed have a complex morphology with many branches (Cattaert et al., 1994b; Clarac and Cattaert, 1996; Watson et al., 2000), and there are few data concerning the location of monosynaptic (Cattaert and El Manira, 1999) and no data about polysynaptic input synapses. Second, the inter-MNs variability 
could suggest an heterogeneity in the MN population. Such a heterogeneity was shown for the response of Dep MNs to acetylcholine (ACh). Some Dep MN are conditional oscillators and present rhythmic oscillations of their membrane potential in the presence of ACh, whereas others never do (Cattaert et al., 1994a,b).

One possible source of inter-individual variability could be related to the social status of the animal. In crayfish, the effect of $5 \mathrm{HT}$ on the neural circuit for tail-flip escape behavior was found to depend also on the animals' social experience. 5HT reversibly enhanced the response to sensory stimuli of the lateral giant tailflip command neuron in socially dominant crayfish and reversibly inhibited it in subordinate animals (Yeh et al., 1996). All of the animals used in our experiments were maintained in large tanks containing many animals. Therefore, we did not know the social status of each experimental animal. Given the fact that dominant animals generally present a more elevated posture than subordinate animals, we hypothesize that 5HT can enhance the resistance reflex response of the Dep MN in dominant animals and have no effect (or a negative effect) on the resistance reflex in subordinate animals. However, because of the fact that $5 \mathrm{HT}$ increases reflex activation in both Dep and Lev MNs, it is likely that if this hypothesis is true, 5HT modulation would lead to an overall increase in joint stiffness rather than toward an asymmetric change in joint posture in dominant animals. These effects are also dependent on which MNs participate to maintaining posture, and therefore the effect of $5 \mathrm{HT}$ on posture in vivo cannot be drawn directly from the present results.

\section{References}

Alvarado-Alvarez R, Arechiga H, Garcia U (2000) Serotonin activates a $\mathrm{Ca}(2+)$-dependent $\mathrm{K}(+)$ current in identified peptidergic neurons from the crayfish. J Exp Biol 203:715-723.

Baines RA, Tyrer NM, Downer RG (1990) Serotoninergic innervation of the locust mandibular closer muscle modulates contractions through the elevation of cyclic adenosine monophosphate. J Comp Neurol 294:623-632.

Beltz BS, Kravitz EA (1983) Mapping of serotonin-like immunoreactivity in the lobster nervous system. J Neurosci 3:585-602.

Cantrell AR, Catterall WA (2001) Neuromodulation of $\mathrm{Na}^{+}$channels: an unexpected form of cellular plasticity. Nat Rev Neurosci 2:397-407.

Cattaert D, El Manira A (1999) Shunting versus inactivation: analysis of presynaptic inhibitory mechanisms in primary afferents of the crayfish. J Neurosci 19:6079-6089.

Cattaert D, Araque A, Buño W, Clarac F (1994a) Motor neurones of the crayfish walking system possess $\mathrm{TEA}^{+}$-revealed regenerative electrical properties. J Exp Biol 188:339-345.

Cattaert D, Araque A, Buño W, Clarac F (1994b) Nicotinic and muscarinic activation of motoneurons in the crayfish locomotor network. J Neurophysiol 72:1622-1633.

Clarac F, Cattaert D (1996) Invertebrate presynaptic inhibition and motor control. Exp Brain Res 112:163-180.

Delaney K, Tank DW, Zucker RS (1991) Presynaptic calcium and serotonin-mediated enhancement of transmitter release at crayfish neuromuscular junction. J Neurosci 11:2631-2643.

Edwards DH, Yeh SR, Musolf BE, Antonsen BL, Krasne FB (2003) Metamodulation of the crayfish escape circuit. Brain Behav Evol 60:360-369.

El Manira A, Cattaert D, Clarac F (1991a) Monosynaptic connections mediate resistance reflex in crayfish (Procambarus clarkii) walking legs. J Comp Physiol [A] 168:337-349.

El Manira A, DiCaprio RA, Cattaert D, Clarac F (1991b) Monosynaptic interjoint reflexes and their central modulation during fictive locomotion in crayfish. Eur J Neurosci 3:1219-1231.

Fischer L, Florey E (1983) Modulation of synaptic transmission and excitation-contraction coupling in the opener muscle of the crayfish, Astacus leptodactylus, by 5-hydroxytryptamine and octopamine. J Exp Biol 102:187-198.
Glusman S, Kravitz EA (1982) The action of serotonin on excitatory nerve terminals in lobster nerve-muscle preparations. J Physiol (Lond) 325: 223-241.

Hasuo H, Matsuoka T, Akasu T (2002) Activation of presynaptic 5-hydroxytryptamine $2 \mathrm{~A}$ receptors facilitates excitatory synaptic transmission via protein kinase $\mathrm{C}$ in the dorsolateral septal nucleus. J Neurosci 22:7509-7517.

Huber R, Orzeszyna M, Pokorny N, Kravitz EA (1997a) Biogenic amines and aggression: experimental approaches in crustaceans. Brain Behav Evol 50 [Suppl 1]:60-68.

Huber R, Smith K, Delago A, Isaksson K, Kravitz EA (1997b) Serotonin and aggressive motivation in crustaceans: altering the decision to retreat. Proc Natl Acad Sci USA 94:5939-5942.

Huber R, Panksepp JB, Yue Z, Delago A, Moore P (2001) Dynamic interactions of behavior and amine neurochemistry in acquisition and maintenance of social rank in crayfish. Brain Behav Evol 57:271-282.

Hultborn H, Kiehn O (1992) Neuromodulation of vertebrate motor neuron membrane properties. Curr Opin Neurobiol 2:770-775.

Katz PS (1998) Neuromodulation intrinsic to the central pattern generator for escape swimming in Tritonia. Ann NY Acad Sci 860:181-188.

Katz PS, Harris-Warrick RM (1989) Serotonergic/cholinergic muscle receptor cells in the crab stomatogastric nervous system. II. Rapid nicotinic and prolonged modulatory effects on neurons in the stomatogastric ganglion. J Neurophysiol 62:571-581.

Kiehn O, Harris-Warrick RM (1992) Serotonergic stretch receptors induce plateau properties in a crustacean motor neuron by a dual-conductance mechanism. J Neurophysiol 68:485-495.

Kravitz EA (1988) Hormonal control of behavior: amines and the biasing of behavioral ouptput in lobsters. Science 241:1775-1781.

Le Bon-Jego M, Cattaert D (2002) Inhibitory component of the resistance reflex in the locomotor network of the crayfish. J Neurophysiol 88:2575-2588.

Le Ray D, Clarac F, Cattaert D (1997a) Functional analysis of the sensory motor pathway of resistance reflex in crayfish. I. Multisensory coding and motor neuron monosynaptic responses. J Neurophysiol 78:3133-3143.

Le Ray D, Clarac F, Cattaert D (1997b) Functional analysis of the sensory motor pathway of resistance reflex in crayfish. II. Integration of sensory inputs in motor neurons. J Neurophysiol 78:3144-3153.

Livingstone MS, Harris-Warrick RM, Kravitz EA (1980) Serotonin and octopamine produce opposite postures in lobsters. Science 208:76-79.

Marder E, Thirumalai V (2002) Cellular, synaptic and network effects of neuromodulation. Neural Netw 15:479-493.

McCormick DA, Pape HC (1990) Noradrenergic and serotonergic modulation of a hyperpolarization-activated cation current in thalamic relay neurones. J Physiol (Lond) 431:319-342.

Nagayama T (2002) Serotonergic modulation of nonspiking local interneurones in the terminal abdominal ganglion of the crayfish. J Exp Biol 205:3067-3076.

Nusbaum MP, Blitz DM, Swensen AM, Wood D, Marder E (2001) The roles of co-transmission in neural network modulation. Trends Neurosci 24:146-154.

Pearlstein E, Clarac F, Cattaert D (1998) Neuromodulation of reciprocal glutamatergic inhibition between antagonistic motoneurons by 5-hydroxytryptamine (5HT) in crayfish walking system. Neurosci Lett 241:37-40.

Qian SM, Delaney KR (1997) Neuromodulation of activity-dependent synaptic enhancement at crayfish neuromuscular junction. Brain Res 771:259-270.

Rasmussen K, Aghajanian GK (1990) Serotonin excitation of facial motoneurons: receptor subtype characterization. Synapse 5:324-332.

Rossi-Durand C (1993) Peripheral proprioceptive modulation in crayfish walking leg by serotonin. Brain Res 632:1-15.

Sheldon PW, Aghajanian GK (1991) Excitatory responses to serotonin (5HT) in neurons of the rat piriform cortex: evidence for mediation by $5 \mathrm{HT} 1 \mathrm{C}$ receptors in pyramidal cells and 5HT2 receptors in interneurons. Synapse 9:208-218.

Sillar KT, Skorupski P (1986) Central input to primary afferent neurons in crayfish, Pacifastacus leniusculus, is correlated with rhythmic motor output of thoracic ganglia. J Neurophysiol 55:678-688.

Sillar KT, Reith CA, McDearmid JR (1998) Development and aminergic 
neuromodulation of a spinal locomotor network controlling swimming in Xenopus larvae. Ann NY Acad Sci 860:318-332.

Southard RC, Haggard J, Crider ME, Whiteheart SW, Cooper RL (2000) Influence of serotonin on the kinetics of vesicular release. Brain Res 871:16-28.

Teshiba T, Shamsian A, Yashar B, Yeh SR, Edwards DH, Krasne FB (2001) Dual and opposing modulatory effects of serotonin on crayfish lateral giant escape command neurons. J Neurosci 21:4523-4529.

Tierney AJ, Mangiamele LA (2001) Effects of serotonin and serotonin analogs on posture and agonistic behavior in crayfish. J Comp Physiol [A] 187:757-767.

Van Dongen PA, Grillner S, Hokfelt T (1986) 5-Hydroxytryptamine (sero- tonin) causes a reduction in the afterhyperpolarization following the action potential in lamprey motoneurons and premotor interneurons. Brain Res 366:320-325.

Wang C, Zucker RS (1998) Regulation of synaptic vesicle recycling by calcium and serotonin. Neuron 21:155-167.

Watson AHD, Bevengut M, Pearlstein E, Cattaert D (2000) GABA and glutamate-like immunoreactivity at synapses on depressor motorneurones of the leg of the crayfish, Procambarus clarkii. J Comp Neurol 422:510-520.

Yeh SR, Fricke RA, Edwards DH (1996) The effect of social experience on serotonergic modulation of the escape circuit of crayfish. Science 271: $366-369$. 